\title{
A Structure-based Virtual Screening Protocol for in silico Identification of Potential Thyroid Disrupting Chemicals Targeting Transthyretin
}

\section{(Supporting Information)}

Jin Zhang $\dagger$, Afshan Begum $\dagger$, Kristoffer Brännström $\dagger$,Christin Grundström $\dagger$, Irina Iakovleva $\downarrow$, Anders Olofsson $\ddagger$, A. Elisabeth Sauer-Eriksson †, Patrik L. Andersson †*

†Department of Chemistry, Umeå University, SE-901 87 Umeå, Sweden

†Department of Medical Biochemistry and Biophysics, Umeå University, SE-901 87 Umeå, Sweden

\section{Corresponding Author}

*Email: patrik.andersson@umu.se. Phone no: +46-90-786-5266, Fax: +46-90-786-7655 
The supporting information (50 pages) contains the following sections:

\section{Contents}

Reasons for the activity cutoff setting

MD simulations

MM/GBSA binding free energy calculations

MM-GBSA energy decomposition

Experimental procedures of the human plasma assay

Potential reasons for the non-active tested compounds.

Environmental implications of the three herbicides identified as TTR binders

Table S1. Data collection and refinement statistics for the TTR-THDCs complex structures

Table S2. Data collection and refinement statistics for the TTR complex structures with DNPC, clonixin, BPS and triclopyr.

Table S3. Ligand binding free energy of BP2, PFOA, and PFOS

Table S4. Collections of TTR binders used for benchmarking set

Table S5. Results of the molecular docking

Table S6. Potential TTR binders proposed by the developed virtual screening protocol

Table S7. Information of the previously confirmed TTR binders in the potential TTR binders

Table S8. Information of the industrial compounds tested by ITC and human plasma assay

Table S9. Thermodynamic parameters of the active compounds and T4 binding to TTR.

Figure S1. Comparisons of binding conformations between BP2, PFOA, PFOS, and T4.

Figure S2. Close-up views of the interactions between TTR and BP2, PFOA, and PFOS

Figure S4. Comparison of the backbones and ligand poses between the crystal structures and MD-averaged structures

Figure S5. Correlations between the calculated binding free energies and the potencies of BP2, PFOA, and TBBPA.

Figure S6. The receiver operating characteristic curves of molecular docking models

Figure S8. Correlations between the docking scores and the binding affinities of the active compounds.

Figure S9. Structures and chemical information of the five false positives identified by ITC. 
Reasons for the activity cutoff setting. The activity $\left(\mathrm{IC}_{50}\right.$ or $\left.\mathrm{K}_{\mathrm{i}}\right)$ cutoff criterion of $100 \mu \mathrm{M}$ was chosen considering the following reasons:

(1) The cutoff value of $100 \mu \mathrm{M}$ was used to define active compounds for biological targets in the Tox 21 program such as the estrogen receptor $^{1,2}$ and the thyroid receptor ${ }^{3}$, and it was also used in the "Tox21Challenge in silico model-developing exercise" initiated by US EPA for identifying potential hazardous compounds. ${ }^{4}$

(2) The $100 \mu \mathrm{M}$ criterion was also set to reach a precautionary identification of emerging industrial compounds with potential human health impacts. Compounds of concern in the benchmarking set, includes propyl paraben $\left(\mathrm{IC}_{50}=19 \mu \mathrm{M}\right)$, butyl paraben $\left(\mathrm{IC}_{50}=43 \mu \mathrm{M}\right)$, and 2,6-dichlorophenol $\left(\mathrm{IC}_{50}=22 \mu \mathrm{M}\right)$, and we wanted to be able to find chemicals in a similar potency range. 
MD simulations. The systems for MD simulations were prepared based on the three structures of TTR complexes with BP2, PFOA (sharing similar binding profiles to PFOS), and TBBPA (PDB ID: $5 \mathrm{HJG})^{7}$,respectively. For each system, hydrogen atoms were added to the TTR structure, followed by optimization of hydrogen bonding networks, and adjusting the protonation state of each residue to its corresponding protonation state at $\mathrm{pH}$ 7.0. The protonation forms of co-ligands were determined considering both their protonation states at $\mathrm{pH} 7.0 \pm 1.0$ and the pronation forms of the nearby residues. These preparation steps were performed using the Schrödinger Protein Preparation Wizard. ${ }^{8}$ The system was solvated in a truncated octahedral water box with $10 \AA$ buffer space and counter ions $\left(\mathrm{Na}^{+}\right.$or $\left.\mathrm{Cl}^{-}\right)$were added to neutralize the system. The TTR protein, the three THDCs, and water molecules were presented using Amber99SB force field, GAFF force field, and TIP3P model, respectively. The partial atomic charges of the co-ligands were calculated at HF/6-31G* level using GAUSSIAN 09.

The MD simulations of the three prepared complex systems (TTR-BP2, TTR-PFOA, and TTR-TBBPA) were performed using Amber $14^{10}$ following the steps below. In the first step, two-stage energy minimizations were applied to remove the poor contacts and relax each system. In the first stage, 1000 cycles of steepest descent and 2000 cycles of conjugate gradient minimizations were applied with the protein backbone restrained $\left(50 \mathrm{kcal} / \mathrm{mol}^{*} \AA 2\right)$ to homogenize the solvents. In the second stage, the whole system was minimized without any restraints (1500 cycles of steepest descent and 3500 cycles of conjugate gradient minimizations). In the second step, each system was gradually heated from $0 \mathrm{~K}$ to $310 \mathrm{~K}$ over 200 ps followed by 500 ps initial equilibration at constant volume and temperature at $310 \mathrm{~K}$. Next, an additional $1 \mathrm{~ns}$ equilibration was performed at constant pressure and temperature (NPT ensemble; $310 \mathrm{~K}, 1 \mathrm{bar}$ ) at 2 ps time steps. Finally, the 20 ns production MD simulations were performed under NPT conditions with Langevin thermostat and Berdensson barostat. All bonds involving hydrogens were constrained with SHAKE. The time step for MD simulation was 2 fs and the integrator leap-frog algorithm was employed. Van der Waals and short-range electrostatic interactions were cut off at $10 \AA$. Long-range electrostatic interactions were computed by the particle mesh Ewald summation (PME) method. ${ }^{11}$ During the sampling process, the coordinates of each complex were saved every 20 ps. 
MM/GBSA binding free energy calculations. We calculated the binding free energies of BP2, PFOA, and TBBPA (single orientation of co-ligands in the TBS situated at the BB' interface) to TTR using the MM-GBSA method in AmberTools $15^{10}$ based on the 1000 snapshots evenly extracted from the stable 10-20 ns MD simulation trajectory. The equations used in the calculations were shown as follows:

$$
\begin{gathered}
\Delta G_{\text {bind }}=\Delta H-T \Delta S \approx \Delta E_{M M}+\Delta G_{\text {Sol }}-T \Delta S \\
\Delta E_{M M}=\Delta E_{\text {Internal }}+\Delta E_{\text {Electro }}+\Delta E_{v d W} \\
\Delta G_{\text {Sol }}=\Delta G_{G B}+\Delta G_{S A}
\end{gathered}
$$

where $\Delta \mathrm{E}_{\mathrm{MM}}$ is the gas-phase interaction energy between the receptor and the ligand, which consists of the internal energy $\left(\Delta \mathrm{E}_{\text {Internal }}\right)$, electrostatic $\left(\Delta \mathrm{G}_{\text {Electro }}\right)$ and van der Waals $\left(\Delta \mathrm{E}_{\mathrm{vdW}}\right)$ terms but $\Delta \mathrm{E}_{\text {Internal }}$ is canceled by using the single-trajectory approach. The solvation free energy $\left(\Delta \mathrm{G}_{\mathrm{Sol}}\right)$ is composed of the polar $\left(\Delta \mathrm{G}_{\mathrm{GB}}\right)$ and non-polar $\left(\Delta \mathrm{G}_{\mathrm{SA}}\right)$ contributions. The entropy term $(\Delta \mathrm{S})$ was neglected due to the high computational cost and low accuracy. The van der Waals $\left(\Delta \mathrm{E}_{\mathrm{vdW}}\right)$ and electrostatic $\left(\Delta \mathrm{G}_{\text {Electro }}\right)$ terms were calculated using the sander module in AMBER14. ${ }^{10}$ The polar solvation was calculated by the GB model developed by Onufriev et al $(\mathrm{igb}=2),{ }^{12}$ whereas the non-polar solvation was estimated by the solvent accessible surface area computed from the LCPO method. ${ }^{13}$ The solute dielectric constant (ein) was set to 1 , and the solvent dielectric constant was set to 80 . 
MM-GBSA energy decomposition. To explore the contributions of each residue to the ligand-TTR binding, the binding free energy of the three coligands (BP2, PFOA and TBBPA) were decomposed onto the each residue in the thyroxine binding site (TBS) using the MM-GBSA pairwise decomposition (idecomp = 3) analysis in AmberTool 15. The total ligand binding free energy of each coligand was decomposed into four components: van der Waals contribution $\left(\Delta \mathrm{G}_{\mathrm{vdW}}\right)$, electrostatic contribution $\left(\Delta \mathrm{G}_{\text {Electro }}\right)$, polar solvation contribution $\left(\Delta \mathrm{G}_{\mathrm{GB}}\right)$ and non-polar solvation contribution $\left(\Delta \mathrm{G}_{\mathrm{SA}}\right)$. The polar part of the solvation free energy $\left(\Delta \mathrm{G}_{\mathrm{GB}}\right)$ was calculated by the GB model with the parameter developed by Onufriev et al $(\mathrm{igb}=2),{ }^{12}$ and the non-polar part $\left(\Delta \mathrm{G}_{\mathrm{SA}}\right)$ was estimated by the SASA computed from the LCPO method. ${ }^{13}$ 
Experimental procedures of the human plasma assay. Specific binding of BP2, PFOS, and PFOA to TTR in the presence of human plasma was measured as previously described. ${ }^{14}$ Briefly, each ligand was titrated into human plasma and incubated for $2 \mathrm{~h}$ under gentle agitation. The conversion of tetrameric TTR to monomer was initiated by the addition of urea to a final concentration of $4.0 \mathrm{M}$. After $18 \mathrm{~h}$ incubation at room temperature, the formation of monomeric TTR was evaluated by gel electrophoresis using tricinebased SDS-PAGE, which prevents the dissociation of tetramers and the re-association of monomers. The monomeric band on the membrane was detected by western blot according to standard protocols using a rabbit anti-TTR antibody (anti-prealbumin, DAKO) followed by a horseradish peroxidase-labeled antibody (anti-rabbit HRP, GE Healthcare). Enhanced chemiluminescence (ECL-prime, GE Healthcare) was used to visualize the bound antibodies. The monomeric bands were quantified by densitometric analysis in the ImageJ 1.48 program. 
Potential reasons for the non-active tested compounds. Five industrial compounds proposed as TTRbinders by the VS protocol showed no activities in the ITC experiments. Some potential reasons for the mispredictions; 2-nitro-5-(2-chloro-4-trifluoromethylphenoxy)benzoic acid was predicted to fit into the TBS with the trifluoromethyl group facing the Ser117 and the acid group interacting with Lys15. However, its ortho-nitrobenzoic acid group can form intramolecular H-bonds that weaken its electrostatic interactions with Lys15 and may prevent its binding with TTR. 2,2'-dihydroxy-4,4'-dimethoxybenzophenone shares the same benzophenone structural motif as BP2. However, the compound has two less hydroxyl groups, which will reduce the number of potential H-bonds between the compound and the polar residues in the TBS, such as Ser117, Lys15 and Thr119, and thus significantly weaken its affinity to TTR. The bulky butanoic acid group of the diphenolic acid greatly increases its molecular volume, which hinder it to fit into the relatively narrow TBS. L- $\gamma$-Glutamyl-p-nitroanilide and 1-(4-sulfophenyl)-3-carboxy-5-pyrazolone have polar acidic groups at both ends of their bulky phenolic structures. The acid groups will greatly decrease their hydrophobicity, which could prevent them from interacting with the hydrophobic residues located in the inner part of the TBS or binding into the relatively hydrophobic TBS. 
Environmental implications of the three herbicides identified as TTR binders. Three more herbicides were confirmed as TTR binders, including fluroxypyr, picloram, and mesotrione.

Fluroxypyr is an herbicide used to control broadleaf weeds and woody brush. Its exposure has shown to decrease weight of the thyroid gland in female rats. ${ }^{15}$ The compound was detected in soil samples from China and Sweden, ${ }^{16,17}$ and the local groundwater in Sweden and France. ${ }^{18,19}$ It was eventually ingested by fish and accumulated in the muscle tissue. ${ }^{19}$

Picloram is used as replacement to 2,4,5-T for general woody plant control ${ }^{20}$. It has been classified as a persistent herbicide. ${ }^{21}$ Exposure to picloram may lead to chronic toxicity in fish. ${ }^{22}$ and development of carcinomas in the thyroid gland and reproductive organs in rats ${ }^{23}$. The compound was detected in the soil samples from China ${ }^{24}$ and $\mathrm{USA}^{25}$; and in the groundwater in Australia. ${ }^{26}$

Mesotrione is an emerging herbicide to replace atrazine in the EU for removing annual grasses and broadleaved weeds in maize. ${ }^{27}$ The compound could alter the metabolism rates in fish, ${ }^{28}$ and induce thyroid proliferations as well as developmental disorders in rats. ${ }^{29}$ Here we suggest TTR as one of its potential targets of its thyroid disruptions. It was detected in soil samples from open field in China, ${ }^{30}$ and in groundwater samples from Poland and Italy. ${ }^{31,32}$ 
Table S1. Data collection and refinement statistics for the TTR complex structures with BP2, PFOA, and PFOS.

\begin{tabular}{|c|c|c|c|}
\hline Data-collection statistics & TTRwt-PFOA & TTRwt-PFOS & TTRwt-BP2 \\
\hline ESRF Beamline $^{\mathrm{a}}$ & ID29 & ID29 & ID29 \\
\hline Wavelength $(\AA)$ & 0.913 & 0.913 & 0.913 \\
\hline Space group & $\mathrm{P} 21212$ & $\mathrm{P} 21212$ & $\mathrm{P} 21212$ \\
\hline Unit-cell parameters $(\AA)$ & $\begin{array}{l}a=42.6 \\
b=85.7 \\
c=63.4\end{array}$ & $\begin{array}{l}a=43.1 \\
b=85.4 \\
c=64.1\end{array}$ & $\begin{array}{l}a=42.9 \\
b=85.5 \\
c=64.2\end{array}$ \\
\hline Resolution limits $(\AA)$ & $\begin{array}{l}40.0-1.22 \\
(1.24-1.20)\end{array}$ & $\begin{array}{l}40.0-1.26 \\
(1.30-1.26)\end{array}$ & $\begin{array}{l}40.0-1.45 \\
(1.50-1.45)\end{array}$ \\
\hline Total No. of reflections & 610176 & 461534 & 301475 \\
\hline No. of unique reflections & $71054(5468)$ & $64626(6308)$ & $42623(4024)$ \\
\hline Multiplicity & $8.6(6.1)$ & $7.1(6.5)$ & $7.1(6.9)$ \\
\hline Completeness (\%) & $96.58(75.14)$ & $99.76(98.83)$ & $99.40(94.90)$ \\
\hline $\mathrm{R}_{\mathrm{sym}}$ & $0.048(0.787)$ & $0.036(0.565)$ & $0.039(0.676)$ \\
\hline$<\mathrm{I} / \sigma(\mathrm{I})\rangle$ & $19.26(1.79)$ & $23.41(2.97)$ & $23.35(2.74)$ \\
\hline \multicolumn{4}{|l|}{ Refinement and model building statistics } \\
\hline Resolution Range $(\AA)$ & $40.0-1.22$ & $40.0-1.26$ & $40.0-1.45$ \\
\hline $\mathrm{R}$ factor $(\%)$ & $14.24(22.25)$ & $13.45(17.53)$ & $13.14(19.55)$ \\
\hline $\mathrm{R}$ free $(\%)$ & $16.32(23.96)$ & $15.83(20.44)$ & $16.77(28.18)$ \\
\hline No. of protein atoms & 2234 & 1962 & 1988 \\
\hline No. of water molecules & 210 & 209 & 240 \\
\hline No. of ligand atoms /glycerol atoms /sodium ion & $50 / 0 / 1$ & $58 / 0 / 1$ & $36 / 12 / 1$ \\
\hline \multicolumn{4}{|l|}{ Rms deviations from ideal geometry } \\
\hline Bond length $(\AA)$ & 0.009 & 0.009 & 0.009 \\
\hline Bond angles $\left({ }^{\circ}\right)$ & 1.35 & 1.36 & 1.22 \\
\hline \multicolumn{4}{|l|}{ Ramachandran plot } \\
\hline Residues in most favored regions (\%) & 98.0 & 98 & 98 \\
\hline Residues allowed regions (\%) & 2.0 & 2 & 2 \\
\hline Residues in disallowed regions (\%) & 0.0 & 0.0 & 0.0 \\
\hline Average B-factor $\left(\AA^{2}\right)$ & 23.5 & 24.8 & 27.5 \\
\hline PDB code & $5 \mathrm{JID}$ & 5JIM & $5 \mathrm{JIQ}$ \\
\hline
\end{tabular}

Note: Values in parenthesis are for the high resolution shell. ${ }^{\text {a }}$ The diffraction data were processed with $\mathrm{XDS}^{33}$ and scaled with AIMLESS from the CCP4 software suite. ${ }^{34}$ 
Table S2. Data collection and refinement statistics for the TTR complex structures with DNPC, clonixin,

BPS and triclopyr.

\begin{tabular}{|c|c|c|c|c|}
\hline Data-collection statistics & \begin{tabular}{|l|} 
TTRwt-DNPC \\
\end{tabular} & TTRwt-clonixin & TTRwt-BPS & TTRwt-triclopyr \\
\hline ESRF Beamline/In-house & ID23-1 & ID23-1 & ID23-1 & In-house $^{\mathrm{a}}$ \\
\hline Wavelength $(\AA)$ & 0.873 & 0.873 & 0.873 & 1.542 \\
\hline Space group & $\mathrm{P} 22_{1} 22$ & $\mathrm{P} 22_{1} 2$ & $\mathrm{P} 2_{1} 22_{1}$ & $\mathrm{P} 22_{1} 2$ \\
\hline Unit-cell parameters $(\AA)$ & $43.2,85.7,65.0$ & $43.1,86.3,64.3$ & $42.8,86.2,64.6$ & $42.5,85.5,64.0$ \\
\hline Resolution limits $(\AA)$ & \begin{tabular}{|l|}
$42.9-1.48$ \\
$(1.53-1.48)$
\end{tabular} & $\begin{array}{l}43.1-1.45 \\
(1.47-1.45)\end{array}$ & $\begin{array}{l}43.1-1.62 \\
(1.68-1.62)\end{array}$ & $\begin{array}{l}27.3-1.58 \\
(1.68-1.58)\end{array}$ \\
\hline Total No. of reflections & \begin{tabular}{|l|}
269102 \\
$(25577)$
\end{tabular} & 269881 & $201068(18921)$ & 101969 \\
\hline No. of unique reflections & 40934(4016) & $43302(2116)$ & $31119(3003)$ & $31035(4666)$ \\
\hline Multiplicity & \begin{tabular}{|l|}
$6.6(6.5)$ \\
\end{tabular} & $6.2(6.2)$ & $6.5(6.3)$ & $3.1(2.8)$ \\
\hline Completeness (\%) & $100.0(100.0)$ & $100.0(100.0)$ & $99.9(99.5)$ & $94.8(87.4)$ \\
\hline Rsym & $0.098(1.171)$ & $0.068(1.135)$ & $0.079(1.114)$ & $0.081(0.691)$ \\
\hline$<\mathrm{I} / \sigma(\mathrm{I})>$ & $9.1(1.4)$ & $12.8(1.4)$ & $11.9(1.5)$ & $9.4(1.4)$ \\
\hline \multicolumn{5}{|c|}{ Refinement and model building statistics } \\
\hline Resolution Range $(\AA)$ & \begin{tabular}{|l|}
$42.9-1.48$ \\
$(1.53-1.48)$ \\
\end{tabular} & $\begin{array}{l}35.8-1.45 \\
(1.50-1.45)\end{array}$ & $\begin{array}{l}43.1-1.62 \\
(1.68-1.62)\end{array}$ & $\begin{array}{l}25.6-1.58 \\
(1.63-1.58)\end{array}$ \\
\hline $\mathrm{R}$ factor $(\%)$ & $17.03(28.89)$ & $14.32(21.47)$ & $17.47(27.22)$ & $16.3(33.9)$ \\
\hline $\mathrm{R}$ free $(\%)$ & $19.43(30.84)$ & $18.04(26.47)$ & $21.61(29.21)$ & $20.1(35.8)$ \\
\hline No. of protein atoms ${ }^{b}$ & 1906 & 1905 & 1963 & 1933 \\
\hline No. of water molecules & 209 & 274 & 192 & 204 \\
\hline $\begin{array}{l}\text { No. of ligand atoms } \\
\text { /sodium ion }\end{array}$ & $56 / 1$ & $36 / 1$ & $34 / 1$ & $28 / 1$ \\
\hline \multicolumn{5}{|c|}{ Rms deviations from ideal geometry } \\
\hline Bond length $(\AA)$ & 0.008 & 0.009 & 0.003 & 0.007 \\
\hline Bond angles $\left({ }^{\circ}\right)$ & 0.94 & 0.95 & 0.56 & 0.80 \\
\hline \multicolumn{5}{|l|}{ Ramachandran plot } \\
\hline $\begin{array}{l}\text { Residues in most favored } \\
\text { regions }(\%)\end{array}$ & 98 & 99 & 99 & 98 \\
\hline $\begin{array}{l}\text { Residues allowed regions } \\
(\%)\end{array}$ & 2 & 1 & 1 & 2 \\
\hline $\begin{array}{l}\text { Residues in disallowed } \\
\text { regions }(\%)\end{array}$ & 0 & 0 & 0 & 0 \\
\hline Average B-factor $\left(\AA^{2}\right)$ & 26.1 & 23.8 & 27.2 & 22.6 \\
\hline PDB code & $5 \mathrm{~L} 4 \mathrm{~F}$ & 5L4I & $5 \mathrm{~L} 4 \mathrm{~J}$ & 5L4M \\
\hline
\end{tabular}

Note: Values in parenthesis are for the high resolution shell. ${ }^{\text {a }}$ The diffraction data set of the TTRtriclopyr complex was collected at a wavelength of $0.15418 \mathrm{~nm}(\mathrm{Cu}-\mathrm{K} \alpha$ radiation) on an in-house $\mathrm{X} 8$ Proteum system equipped with a MicroStar-H X-ray generator (Bruker AXS). Diffraction data were integrated with SAINT and scaled using SADAPS (Bruker AXS software suite). ${ }^{\text {b}}$ The variation in the number of refined protein atoms is due to multiple conformations of some residues. 
Table S3. Ligand binding free energy of (a) BP2, (b) PFOA, and (c) TBBPA calculated and decomposed using MM-GBSA method. The protonation form of the compounds used in MD simulations is shower as the structure. The unit of the each term is in $\mathrm{kcal} / \mathrm{mol}$.

a. BP2 (Total binding free energy $=-33.14)$

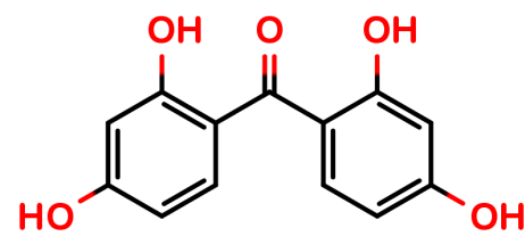

\begin{tabular}{|l|l|l|l|l|l|}
\hline Key Resides & van der Waals & Electrostatic & Polar Solvation & Non-Polar Solvation & TOTAL \\
\hline B-Thr119 & -1.21 & -2.37 & 0.27 & -0.93 & -4.23 \\
\hline B-Ser117 & -0.16 & -2.97 & -0.10 & -0.64 & -3.86 \\
\hline B-Leu110 & -2.15 & 0.13 & -0.02 & -1.28 & -3.34 \\
\hline B-Lys15 & -1.13 & 1.25 & -1.52 & -0.97 & -2.36 \\
\hline B'-Ser117 & -0.20 & -1.17 & -0.22 & -0.53 & -2.12 \\
\hline
\end{tabular}

b. PFOA (Total binding free energy $=-15.71$ )

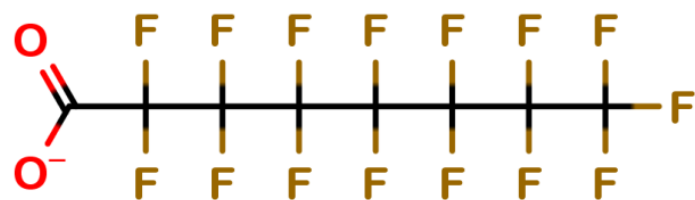

\begin{tabular}{|l|l|l|l|l|l|}
\hline Key Residues & van der Waals & Electrostatic & Polar Solvation & Non-Polar Solvation & TOTAL \\
\hline B-Lys15 & -0.50 & -41.18 & 37.02 & -0.90 & -5.57 \\
\hline B'-Lys15 & -0.92 & -33.91 & 32.50 & -0.91 & -3.24 \\
\hline B-Leu17 & -0.95 & -0.69 & 0.58 & -0.82 & -1.87 \\
\hline B'-Leu17 & -0.91 & -0.51 & 0.41 & -0.82 & -1.82 \\
\hline B-Leu110 & -0.92 & -0.19 & 0.15 & -0.76 & -1.72 \\
\hline
\end{tabular}

c. TBBPA (Total binding free energy $=-46.34$ )

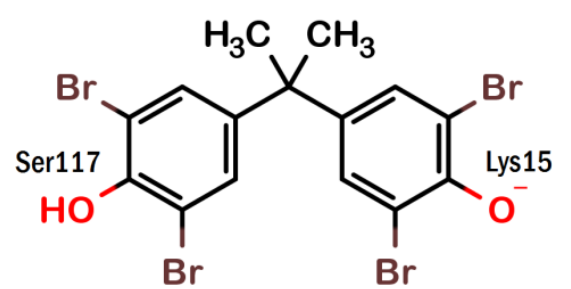

\begin{tabular}{|l|l|l|l|l|l|}
\hline Key Residues & van der Waals & Electrostatic & Polar Solvation & Non-Polar Solvation & TOTAL \\
\hline B-Lys15 & -0.68 & -44.71 & 38.38 & -1.04 & -8.06 \\
\hline B'-Lys15 & -2.53 & -31.22 & 29.39 & -1.84 & -6.20 \\
\hline B-Thr119 & -1.20 & -1.51 & -0.15 & -0.61 & -3.47 \\
\hline B-Leu110 & -1.69 & -0.34 & 0.31 & -0.93 & -2.66 \\
\hline B-Ala108 & -1.51 & -0.57 & 0.64 & -1.06 & -2.50 \\
\hline
\end{tabular}


Table S4. Collections of TTR binders (IC50 or $\mathrm{Ki} \leq 100 \mu \mathrm{M}$ ) used for benchmarking set generation following the Directory of Useful Decoys, Enhanced (DUD-E) guidelines. ${ }^{35}$ The Binders were retrieved from scientific literature and public databases. ${ }^{5,6,36-41}$ For each compound, the protonation form with a more negative docking score (considering both binding affinity and tautomer ratio) was shown as below.<smiles>CC(C)(c1cc(Br)c(OCC(Br)CBr)c(Br)c1)c1cc(Br)c(OCC(Br)CBr)c(Br)c1</smiles>

ChemID: 2-2-Bis[3-5-dibromo-4-(2-3dibromopropoxy)phenyl]propane pKa1: NA

pKa2: NA

Molecular charge: 0<smiles>Oc1ccc(Br)cc1Br</smiles>

ChemID: 2-4-Dibromphenol pKa1: 7.71

pKa2: NA

Molecular charge: 0<smiles>Cc1cc(/C=C/c2ccccc2)cc(C)c1O</smiles>

ChemID: 2-6-Dimethyl-4-styryl-phenol pKa1: 9.72

pKa2: NA

Molecular charge: 0<smiles>Cc1cc(/C=C/c2cccc(C(=O)[O-])c2)cc(C)c1O</smiles>

ChemID: 3-(4-hydroxy-3-5dimethylstyryl)benzoic-acid

pKa1: 4.01

pKa2: 9.72

Molecular charge: -1

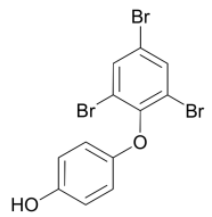

ChemID: 4-(2-4-6-Tribromo-phenoxy)phenol

pKa1: 9.67

pKa2: NA

Molecular charge: 0<smiles>[O-]c1cc(Cl)c(Cl)cc1Cl</smiles>

ChemID: 2-4-5-Trichlorophenol pKa1: 6.83

pKa2: NA

Molecular charge: -1<smiles>O=[N+]([O-])c1ccc([O-])c([N+](=O)[O-])c1</smiles>

ChemID: 2-4-Dinitrophenol pKa1: 4.35

pKa2: NA

Molecular charge: -1<smiles>[O-]c1ccc(Oc2c(Br)cc(Br)cc2Br)cc1Br</smiles>

ChemID: 2-Bromo-4-(2-4-6-tribromophenoxy)-phenol pKa1: 8.29

pKa2: NA

Molecular charge: -1<smiles>O=c1[n-]c(Cl)c(Cl)cc1Cl</smiles>

ChemID: 3,5,6-Trichloro-2-pyridinol pKa1: 8.92

pKa2: NA

Molecular charge: -1

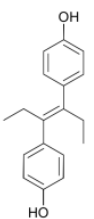

ChemID: 4-4'-(3E)-hex-3-ene-3-4diyldiphenol

pKa1: 8.63

pKa2: 9.23

Molecular charge: 0<smiles>[O-]c1c(Br)cc(Br)cc1Br</smiles>

ChemID: 2-4-6-Tribromphenol pKa1: 6.34

pKa2: NA

Molecular charge: -1<smiles>[O-]c1cc2oc3c(Cl)c(Cl)c(Cl)cc3c2cc1Cl</smiles>

ChemID: 2-6-7-8-tetrachloro-3Dibenzofuranol pKa1: 7.13

pKa2: NA

Molecular charge: -1<smiles>[O-]c1c(Cl)cc2c(c1Cl)Oc1cc(Cl)c(Cl)cc1O2</smiles>

ChemID: 2-Hydroxy-1-3-7-8tetrachlorodibenzo-p-dioxin pKa1: 6.42

pKa2: NA

Molecular charge: -1<smiles>CC(C)(c1cc(Cl)c([O-])c(Cl)c1)c1cc(Cl)c([O-])c(Cl)c1</smiles>

ChemID: 3-3'-5-5'-Tetrachlorobisphenol-

pKa1: 6.27

pKa2: 6.88

Molecular charge: -2<smiles>Oc1c(Cl)c(Cl)c(C(Cl)=C(Cl)Cl)c(Cl)c1Cl</smiles>

ChemID: 4OH-Heptachlorostyrene pKa1: 5.01

pKa2: NA

Molecular charge: -1<smiles>[O-]c1c(Cl)cc(Cl)cc1Cl</smiles>

ChemID: 2-4-6-Trichlorophenol pKa1: 5.99

Molecular charge: -1

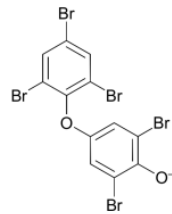

ChemID: 2-6-Dibromo-4-(2-4-6tribromo-phenoxy)-phenol pKa1: 6.93

pKa2: NA

Molecular charge: -1<smiles>Oc1cc2c(cc1Cl)Oc1cc(Cl)c(Cl)cc1O2</smiles>

ChemID: 2-Hydroxy-3-7-8-

trichlorodibenzo-p-dioxin

pKa1: 7.74

pKa2: NA

Molecular charge: 0<smiles>O=[N+]([O-])c1cc([O-])c([O-])c([N+](=O)[O-])c1</smiles>

$\mathrm{HO}$

ChemID: 3-5-Dinitro-benzene-1-2-diol pKa1: 4.74

pKa2: 9.32

Molecular charge: -1<smiles>Oc1ccccc1Oc1ccc(Br)cc1</smiles>

ChemID: 2'-OH-PBDE-3

pKa1: 8.46

pKa2: NA

Molecular charge: 0 
<smiles>Oc1ccccc1Oc1ccc(Br)cc1Br</smiles>

ChemID: 2'-OH-PBDE-7

pKa1: 8.44

pKa2: NA

Molecular charge: 0

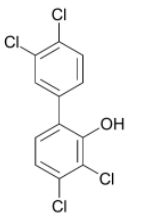

ChemID: 2-OH-PCB-105

pKa1: 7.05

pKa2: NA

Molecular charge: 0<smiles>Oc1cccc(Oc2ccc(Br)cc2Br)c1</smiles>

ChemID: 3'-OH-PBDE-7

pKa1: 9.1

pKa2: NA

Molecular charge: 0<smiles>[O-]c1cc(Oc2ccc(Br)cc2Br)c(Br)cc1Br</smiles>

ChemID: 5-OH-PBDE-47

pKa1: 7.28

pKa2: NA

Molecular charge: -1

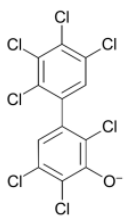

ChemID: 3'-OH-PCB-180

pKa1: 5.71

pKa2: NA

Molecular charge: -1
ChemID: 4'-OH-PBDE-17

pKa1: 9.06

pKa2: NA

Molecular charge: 0

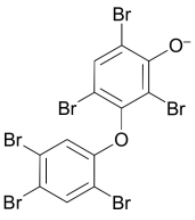

ChemID: 3-OH-PBDE-154

pKa1: 5.94

pKa2: NA

Molecular charge: -1
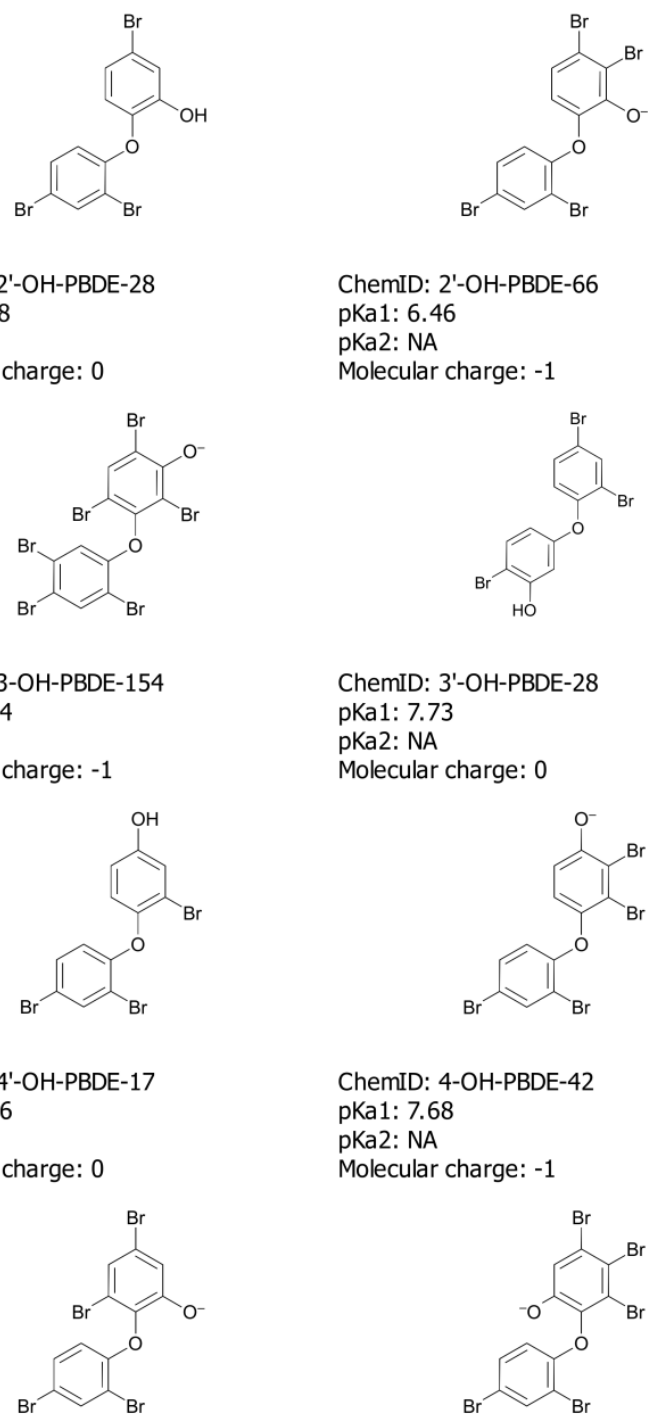

ChemID: 2'-OH-PBDE-66

pKa1: 6.46

pKa2: NA

Molecular charge: -1

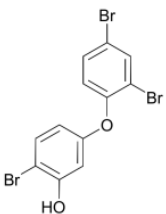

ChemID: 3'-OH-PBDE-28

pKa1: 7.73

pKa2: NA

Molecular charge: 0<smiles>[O-]c1ccc(Oc2ccc(Br)cc2Br)c(Br)c1Br</smiles>

ChemID: 4-OH-PBDE-42

pKa1: 7.68

pKa2: NA

Molecular charge: -1

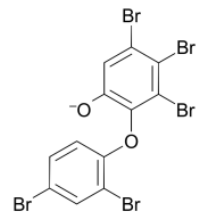

ChemID: 6-OH-PBDE-85 pKa1: 6.71

pKa2: NA<smiles>Oc1c(Cl)cc(Cl)c(-c2cc(Cl)c(Cl)c(Cl)c2Cl)c1Cl</smiles>

ChemID: 3'-OH-PCB-182

pKa1: 5.91

pKa2: NA

Molecular charge: -1

ChemID: 3'-OH-PCB-203

pKa1: 5.67

pKa2: NA

Molecular charge: -1
Molecular charge: -1<smiles>Oc1c(Br)cc(Br)cc1Oc1ccc(Br)cc1Br</smiles>

ChemID: 2'-OH-PBDE-68

pKa1: 6.68

pKa2: NA

Molecular charge: -1<smiles>Oc1c(Br)ccc(Oc2ccc(Br)cc2Br)c1Br</smiles>

ChemID: 3-OH-PBDE-47

pKa1: 6.38

pKa2: NA

Molecular charge: -1<smiles>[O-]c1cc(Br)c(Oc2ccc(Br)cc2Br)cc1Br</smiles>

ChemID: 4'-OH-PBDE-49

pKa1: 7.69

pKa2: NA

Molecular charge: -1<smiles>[O-]c1c(Cl)c(Cl)cc(-c2ccc(Cl)c(Cl)c2Cl)c1Cl</smiles>

ChemID: 3'-OH-PCB-138

pKa1: 5.71

pKa2: NA

Molecular charge: -1<smiles>O=C(O)c1cc(-c2c(Cl)c(Cl)c(Cl)c(Cl)c2Cl)c(Cl)c(Cl)c1Cl</smiles><smiles>COc1c(Cl)cc(-c2cc(Cl)c(O)c(Cl)c2)c(Cl)c1Cl</smiles>

ChemID: 4-4'-OH-PCB-11

pKa1: 5.79

pKa2: 6.52

Molecular charge: -2 


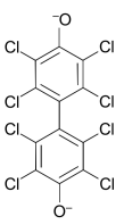

ChemID: 4-4-OH-PCB-202 pKa1: 4.9

pKa2: 5.58

Molecular charge: -2<smiles>O=C(O)c1c(Cl)cc(-c2ccc(Cl)c(Cl)c2Cl)cc1Cl</smiles>

ChemID: 4'-OH-PCB-108

pKa1: 6.27

pKa2: NA

Molecular charge: -1<smiles>[O-]c1c(Cl)cc(-c2cc(Cl)cc(Cl)c2)cc1Cl</smiles>

ChemID: 4-OH-PCB-127

pKa1: 6.37

pKa2: NA

Molecular charge: -1<smiles>Clc1cc(-c2cc(Cl)c(Cl)c(Cl)c2Cl)cc(Cl)c1Cl</smiles>

ChemID: 4'-OH-PCB-172

pKa1: 5.77

pKa2: NA

Molecular charge: -1<smiles>Oc1cc(Cl)c(-c2ccccc2Cl)c(Cl)c1</smiles>

ChemID: 4-OH-PCB-50

pKa1: 8.1

pKa2: NA

Molecular charge: 0
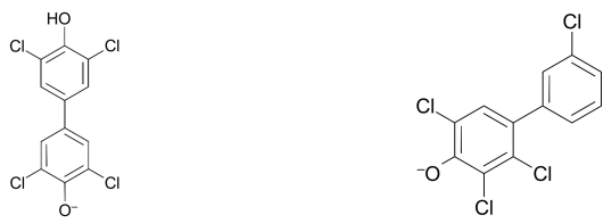

ChemID: 4-4'-OH-PCB-80

pKa1: 6.11

pKa2: 6.79

Molecular charge: -1

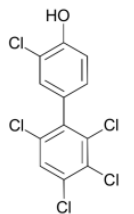

ChemID: 4'-OH-PCB-109

pKa1: 7.6

pKa2: NA

Molecular charge: 0<smiles>Oc1c(Cl)cc(-c2ccc(Cl)c(Cl)c2Cl)c(Cl)c1Cl</smiles>

ChemID: 4'-OH-PCB-130

pKa1: 5.79

pKa2: NA

Molecular charge: -1

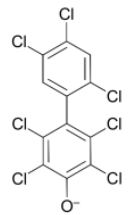

ChemID: 4-OH-PCB-187

pKa1: 4.13

pKa2: NA

Molecular charge: -1

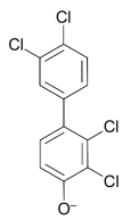

ChemID: 4-OH-PCB-56

pKa1: 7.39

pKa2: NA

Molecular charge: -1
ChemID: 4-OH-PCB-106

pKa1: 5.92

pKa2: NA

Molecular charge: -1<smiles>Oc1c(Cl)c(Cl)c(-c2cccc(Cl)c2)c(Cl)c1Cl</smiles>

ChemID: 4-OH-PCB-112

pKa1: 5.45

pKa2: NA

Molecular charge: -1<smiles>[O-]c1c(Cl)cc(-c2ccccc2)cc1Cl</smiles>

ChemID: 4-OH-PCB-14

pKa1: 6.42

pKa2: NA

Molecular charge: -1<smiles>Oc1c(Cl)c(Cl)c(-c2c(Cl)cc(Cl)c(Cl)c2Cl)c(Cl)c1Cl</smiles>

ChemID: 4-OH-PCB-201

pKa1: 5.18

pKa2: NA

Molecular charge: -1<smiles>Oc1ccc(-c2cc(Cl)c(Cl)c(Cl)c2Cl)cc1</smiles>

ChemID: 4'-OH-PCB-61

pKa1: 9.72

pKa2: NA

Molecular charge: 0

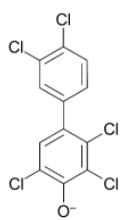

ChemID: 4-OH-PCB-107

pKa1: 5.93

pKa2: NA

Molecular charge: -1<smiles>O=C(O)c1c(Cl)cc(-c2c(Cl)cc(Cl)cc2Cl)cc1Cl</smiles>

ChemID: 4'-OH-PCB-121

pKa1: 6.16

pKa2: NA

Molecular charge: -1<smiles>Oc1c(Cl)cc(-c2cc(Cl)c(Cl)cc2Cl)c(Cl)c1Cl</smiles>

ChemID: 4-OH-PCB-146

pKa1: 5.78

pKa2: NA

Molecular charge: -1

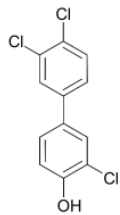

ChemID: 4'-OH-PCB-35

pKa1: 7.85

pKa2: NA

Molecular charge: 0<smiles>Oc1ccc(-c2c(Cl)cc(Cl)cc2Cl)cc1Cl</smiles>

ChemID: 4'-OH-PCB-69

pKa1: 7.61

pKa2: NA

Molecular charge: 0 
<smiles>Oc1c(Cl)cc(-c2ccc(Cl)c(Cl)c2)cc1Cl</smiles>

ChemID: 4'-OH-PCB-79

pKa1: 6.4

pKa2: NA

Molecular charge: -1

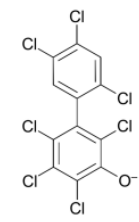

ChemID: 5-OH-PCB-183

pKa1: 5.41

pKa2: NA

Molecular charge: -1

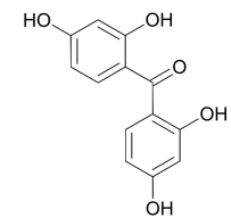

pKa1: 6.75

pKa2: 7.38
ChemID: BP-2

Molecular charge: 0

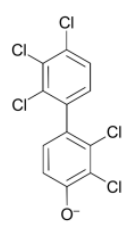

ChemID: 4'-OH-PCB-82

pKa1: 7.25

pKa2: NA

Molecular charge: -1

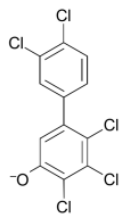

ChemID: 5-OH-PCB-77

pKa1: 6.83

pKa2: NA

Molecular charge: -1<smiles>[O-]c1c(Cl)c(Cl)c(-c2ccccc2Cl)c(Cl)c1Cl</smiles>

ChemID: 4-OH-PCB-93

pKa1: 5.34

pKa2: NA

Molecular charge: -1<smiles>Oc1ccc(/C=C/c2cc(O)cc(O)c2)cc1</smiles>

ChemID: 5-[(E)-2-(4-

hydroxyphenyl)ethenyl]benzene-1-3-

diol

pKa1: 8.49

pKa2: 9.13

Molecular charge: 0<smiles>CCCCOC(=O)c1ccc(O)cc1</smiles><smiles>Cc1cc(/C=C/c2cccc(C(=O)Oc3ccccc3[N+](=O)[O-])c2)cc(C)c1O</smiles><smiles>Cc1cc(/C=C/c2cccc(C(=O)Oc3ccc(F)cc3)c2)cc(C)c1O</smiles>

ChemID: CHEMBL1213030

pKa1: 9.72

pKa2: NA

Molecular charge: 0

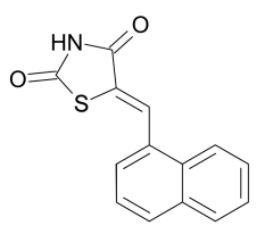

ChemID: CHEMBL1348329

pKa1: 7.9

pKa2: NA

Molecular charge: 0

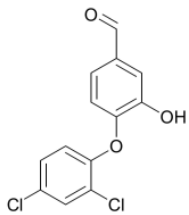

ChemID: CHEMBL1213660

pKa2: NA

Molecular charge: 0

pKa1: 7.86

pKa2: NA

Molecular charge: 0
ChemID: CHEMBL240805

pKa1: 8.32

pKa2: NA

Molecular charge: 0

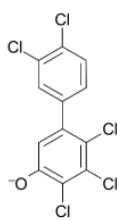

ChemID: 5-OH-PCB-105

pKa1: 6.83

pKa2: NA

Molecular charge: -1<smiles>O=c1cc(-c2ccc(O)cc2)oc2cc(O)cc(O)c12</smiles>

ChemID: Apigenin

pKa1: 6.57

pKa2: 8.07

Molecular charge: 0
ChemID: CHEMBL23588

pKa1: 3.88

pKa2: 17.53

Molecular charge: -1

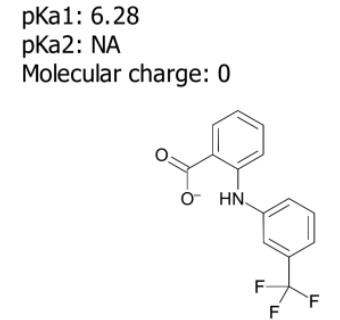

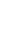

\begin{abstract}
ChemID: Butyl paraben
pKa2: NA

Molecular charge: 0
\end{abstract}

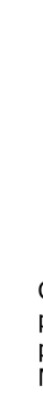

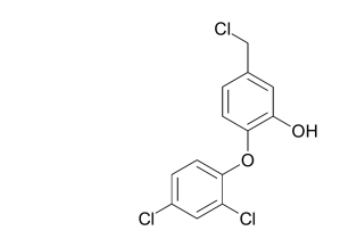

ChemID: CHEMBL1213031

pKa1: 9.72

pKa2: NA

Molecular charge: 0<smiles>COC(=O)c1ccc2nc(-c3cc(Cl)cc(Cl)c3)oc2c1</smiles>

ChemID: CHEMBL2103837

pKa1: 3.6

pKa2: NA

Molecular charge: -1

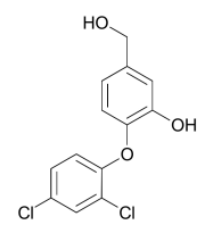

ChemID: CHEMBL240806

pKa1: 8.28

pKa2: 15.15

Molecular charge: 0 
<smiles>COc1cc(CCl)ccc1Oc1ccc(Cl)cc1Cl</smiles>

ChemID: CHEMBL240807

pKa1: NA

pKa2: NA

Molecular charge: 0<smiles>O=C([O-])c1ccc(Oc2ccccc2)c(O)c1</smiles>

\section{ChemID: CHEMBL241454}

pKa1: 4.08

pKa2: 8.44

Molecular charge: -1<smiles>Cc1cc(/C=C/c2cccc(C(=O)O)c2)cc(C)c1O</smiles>

ChemID: CHEMBL442167

pKa1: 4.01

pKa2: 9.72

Molecular charge: -1<smiles>O=C1Nc2ccccc2/C1=N/c1ccc([N+](=O)[O-])cc1</smiles>

ChemID: CHEMBL570013

pKa1: 10.06

pKa2: NA

Molecular charge: 0<smiles>COc1c(Br)cc(/C=C2\SC(=O)NC2=O)cc1Br</smiles>

ChemID: CHEMBL591504

pKa1: 8.1

pKa2: NA

Molecular charge: 0

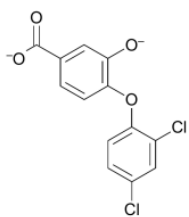

ChemID: CHEMBL240808

pKa1: 4.08

pKa2: 8.43

Molecular charge: -2

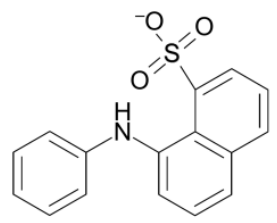

ChemID: CHEMBL285527

pKa1: -2.03

pKa2: 18.91

Molecular charge: -1

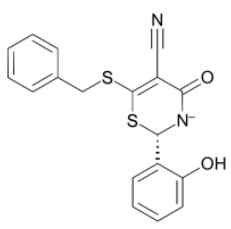

ChemID: CHEMBL493923

pKa1: 9.02

pKa2: 10.2

Molecular charge: -1<smiles>CN1C(=O)/C(=N\c2ccc([N+](=O)[O-])cc2)c2ccccc21</smiles>

ChemID: CHEMBL570239

pKa1: NA

pKa2: NA

Molecular charge: 0<smiles>CN1C(=O)C(=O)c2ccccc21</smiles>

ChemID: CHEMBL60569

pKa1: NA

pKa2: NA

Molecular charge: 0<smiles>COc1cc(CCl)ccc1Oc1ccccc1</smiles>

ChemID: CHEMBL241025

pKa1: NA

pKa2: NA

Molecular charge: 0

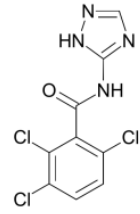

ChemID: CHEMBL394034

pKa1: 8.35

pKa2: 10.57

Molecular charge: 0

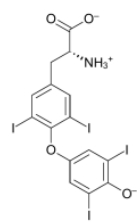

ChemID: CHEMBL559

pKa1: 0.27

pKa2: 7.43

Molecular charge: -1<smiles>Cc1ccc(/N=C2\C(=O)N(C)c3ccccc32)cc1</smiles>

ChemID: CHEMBL570930

pKa1: NA

pKa2: NA

Molecular charge: 0<smiles>[O-]c1c(I)cc(I)cc1I</smiles>

ChemID: CHEMBL81721

pKa1: 6.85

pKa2: NA

Molecular charge: -1<smiles>COc1cc(C=O)ccc1Oc1ccccc1</smiles>

ChemID: CHEMBL241453

pKa1: NA

pKa2: NA

Molecular charge: 0

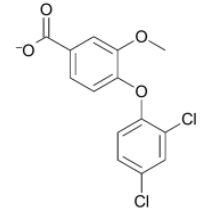

ChemID: CHEMBL438498

pKa1: 4.07

pKa2: NA

Molecular charge: -1<smiles>Cc1cc(/C=C/c2ccccc2)cc(C)c1O</smiles>

ChemID: CHEMBL55960

pKa1: 9.72

pKa2: NA

Molecular charge: 0<smiles>COc1ccc(/N=C2\C(=O)N(C)c3ccccc32)cc1</smiles>

ChemID: CHEMBL576310

pKa1: NA

pKa2: NA

Molecular charge: 0

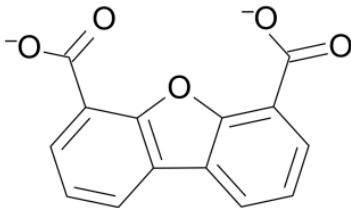

ChemID: Dibenzo[b-d]furan-4-6-

dicarboxylic-acid

pKa1: 3.99

pKa2: 4.6

Molecular charge: -2 


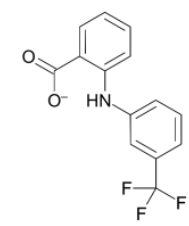

ChemID: Flufenamic-acid

pKa1: 3.88

pKa2: 17.53

Molecular charge: -1

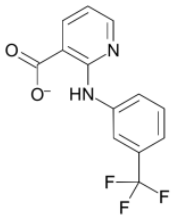

ChemID: Niflumic-acid

pKa1: 1.88

pKa2: 16.44

Molecular charge: -1

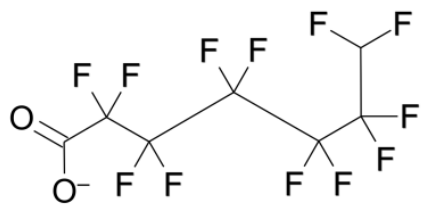

ChemID: DoFHpA

pKa1: -1.36

pKa2: 19.79

Molecular charge: -1

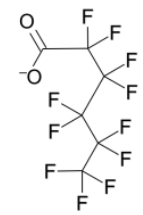

ChemID: Perfluorohexanoic-acid pKa1: -0.78

pKa2: NA

Molecular charge: -1

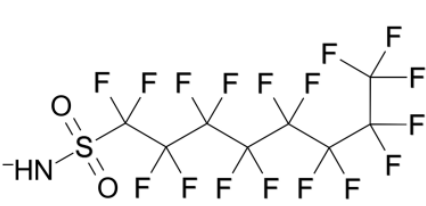

ChemID: perfluorooctane-sulfonamide pKa1: 3.37

pKa2: NA

Molecular charge: -1<smiles>O=c1c(-c2ccc(O)cc2)coc2cc(O)cc(O)c12</smiles>

ChemID: Genistein

pKa1: 6.55

pKa2: 8.09

Molecular charge: 0<smiles>Oc1c(Br)c(Br)c(Br)c(Br)c1Br</smiles>

ChemID: Pentabromphenol

pKa1: 5.01

pKa2: NA

Molecular charge: -1

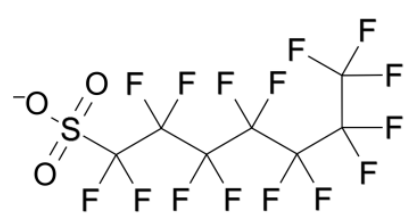

ChemID: Perfluoroheptanesulfonic acid pKa1: -3.32

pKa2: NA

Molecular charge: -1

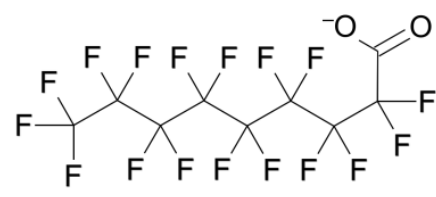

ChemID: Perfluorononanoic-acid pKa1: -6.51

pKa2: NA

Molecular charge: -1

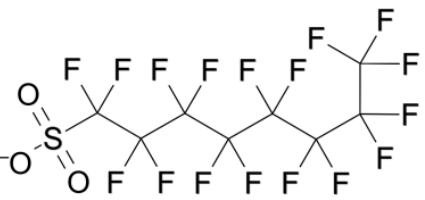

ChemID: Perfluorooctanesulfonic-acid pKa1: -3.32

pKa2: NA

Molecular charge: -1

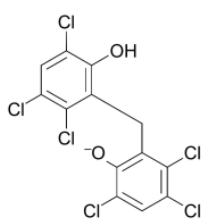

ChemID: Hexachlorophen

pKa1: 5.15

pKa2: 8.79

Molecular charge: -1<smiles>Oc1c(Cl)c(Cl)c(Cl)c(Cl)c1Cl</smiles>

ChemID: Pentachlorophenol pKa1: 4.98

pKa2: NA

Molecular charge: -1

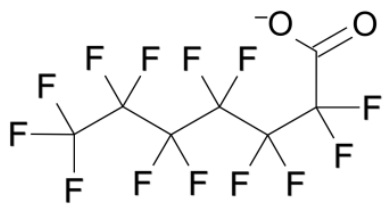

ChemID: Perfluoroheptanoic-acid pKa1: -2.29

pKa2: NA

Molecular charge: -1

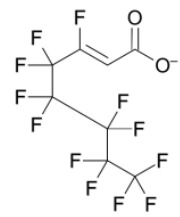

ChemID: 2H-perfluoro-2-octenoic-acid pKa1: -0.46

pKa2: NA

Molecular charge: -1

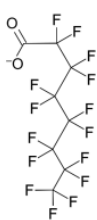

ChemID: Perfluorooctanoic-acid

pKa1: -4.2

pKa2: NA

Molecular charge: -1<smiles>CN1C(=O)C(=O)c2ccccc21</smiles>

ChemID: Isatin-analog-8

pKa1: NA

pKa2: NA

Molecular charge:

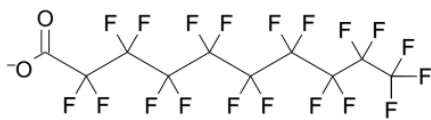

ChemID: perfluorodecanoic-acid

pKa1: -5.2

pKa2: NA

Molecular charge: -1

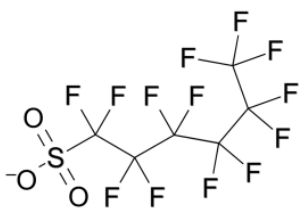

ChemID: Perfluorohexanesulfonic-acid pKa1: -3.32

pKa2: NA

Molecular charge: -1<smiles>O=C([O-])C(F)(F)C(F)(F)C(F)(F)C(F)(F)F</smiles>

ChemID: Perfluoro-n-pentanoic-acid pKa1: 0.34

pKa2: NA

Molecular charge: -1

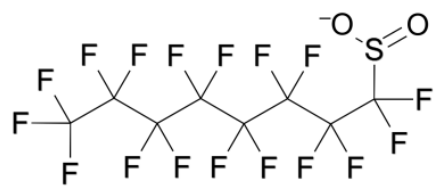

ChemID: Perfluorooctansulfinate

pKa1: 2

pKa2: NA

Molecular charge: -1 
(nFF

ChemID: perfluorotridecanoic-acid pKa1: -5.2

pKa2: NA

Molecular charge: -1<smiles>CC(C)(c1ccc(O)c(Br)c1)c1cc(Br)c([O-])c(Br)c1</smiles>

ChemID: Tribromobisphenol-A

pKa1: 6.86

pKa2: 8.25

Molecular charge: -1

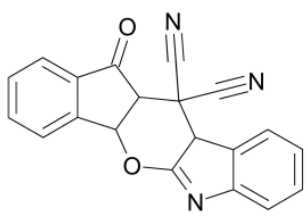

ChemID: US8877795-11

pKa1: 9.04

pKa2: 12.99

Molecular charge: 0

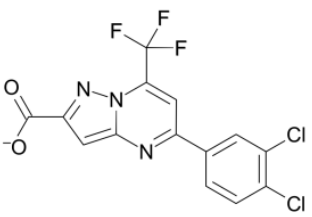

ChemID: US8877795-16

pKa1: 3.12

pKa2: NA

Molecular charge: -1

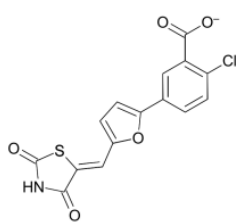

ChemID: US8877795-20

pKa1: 2.94

pKa2: 7.89

Molecular charge: -1
ChemID: Propyl paraben

pKa1: 8.5

pKa2: NA

Molecular charge: 0

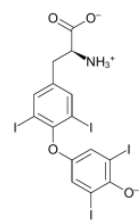

ChemID: T4

pKa1: 0.27

pKa2: 7.43

Molecular charge: -1<smiles>Oc1cc(Cl)ccc1Oc1ccc(Cl)cc1Cl</smiles>

ChemID: Triclosan

pKa1: 7.68

pKa2: NA

Molecular charge: -1<smiles>O=C1NC(=O)/C(=C/c2cccc(C(F)(F)F)c2)S1</smiles>

ChemID: US8877795-12

pKa1: 8.05

pKa2: NA

Molecular charge: 0<smiles>COc1ccc(C(=O)Cc2ccccc2O)c(O)c1</smiles>

ChemID: US8877795-17

pKa1: 7.85

pKa2: 9.82

Molecular charge: -1

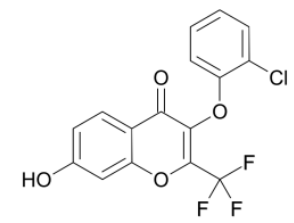

ChemID: US8877795-21

pKa1: 6.29

pKa2: NA

Molecular charge: 0

pKa1: 4.08

pKa2: 8.43

Molecular charge: -2

ChemID: US8877795-14

pKa1: 6.6

pKa2: 10.38

Molecular charge: -1

ChemID: US8877795-18

pKa1: 7.9

pKa2: NA

Molecular charge: 0

ChemID: US8877795-23

pKa1: NA

pKa2: NA

Molecular charge: 0<smiles>CC(C)(c1cc(Br)c([O-])c(Br)c1)c1cc(Br)c(O)c(Br)c1</smiles>

ChemID: Tetrabromo-bisphenol-A pKa1: 6.57

pKa2: 7.18

Molecular charge: -1<smiles>O=C(O)c1ccc(Oc2ccc(Cl)cc2O)c(O)c1</smiles>

ChemID: Triclosan-derivative-7<smiles>CC1(C)NC(=O)C(C#N)=C(SCc2ccccc2)S1</smiles>

ChemID: US8877795-10

pKa1: 10.39

pKa2: NA

Molecular charge: -1<smiles>COc1c(Br)cc(/C=C2/C(=O)N=C(N)N2C)cc1Br</smiles><smiles>Cc1n[nH]c(C)c1CCc1nc2c3ccccc3nc(S)n2n1</smiles>

ChemID: US8877795-15

pKa1: 10.01

pKa2: 16.27

Molecular charge: -1<smiles>Cc1ccccc1/C=C1\SC(=O)NC1=O</smiles><smiles>CSC1=NSC2=NC(=O)/C(=C\c3cccc4ccccc34)C(=N)N12</smiles>

\section{pKa1: 6.33}

ChemID: US8877795-19

pKa2: NA

Molecular charge: 0

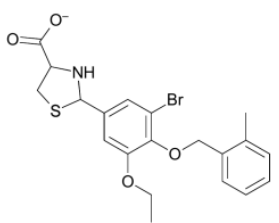

pKa1: 1.78

pKa2: NA

Molecular charge: -1 


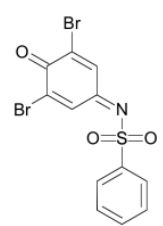

ChemID: US8877795-25

pKa1: NA

pKa2: NA

Molecular charge: 0

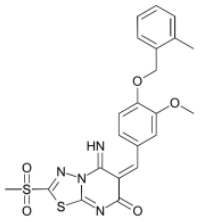

ChemID: US8877795-29

pKa1: NA

pKa2: NA

Molecular charge: 0<smiles>O=C([O-])Cc1ccccc1Nc1c(O)cccc1Cl</smiles>

ChemID: US8877795-Diclofenac

pKa1: 4.03

pKa2: 9.16

Molecular charge: -1

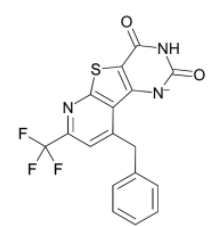

ChemID: US8877795-26

pKa1: 10.98

pKa2: 13.2

Molecular charge: -1

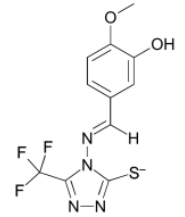

ChemID: US8877795-6

pKa1: 6.55

pKa2: 8.61

Molecular charge: -1

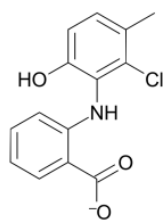<smiles>O=C1NC(=O)/C(=C/c2ccccc2OCc2ccccc2)S1</smiles>

ChemID: US8877795-27

pKa1: 7.9

pKa2: NA

Molecular charge: 0<smiles>Cc1n[nH]c(C)c1CCCOc1ccccc1F</smiles>

ChemID: US8877795-7

pKa1: 16.4

pKa2: NA

Molecular charge: 0

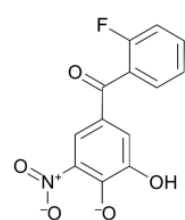

ChemID: US8877795-Meclofenamic-Acid ChemID: US8877795-Ro-41-0960

pKa1: 3.78

pKa2: 9.58

pKa1: 5.39

pKa2: 10.3

Molecular charge: -1

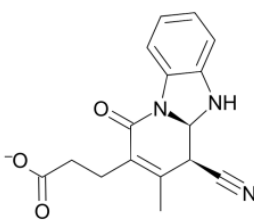

ChemID: US8877795-28

pKa1: 3.08

pKa2: 13.32

Molecular charge: -1

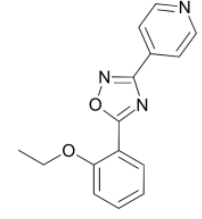

ChemID: US8877795-9

pKa1: NA

pKa2: NA

Molecular charge: 0 
Table S5. Results of the molecular docking under the standard precision.

\begin{tabular}{|c|c|c|c|c|c|}
\hline $\mathrm{PDB} \mathrm{ID}^{\mathrm{a}}$ & No. Active identified ${ }^{b}$ & $\operatorname{RMSD}^{\mathrm{c}}(\AA)$ & AUC & EF10\% & EF20\% \\
\hline TTR-BP2 & 120 & 0.8 & 0.62 & 2.92 & 1.88 \\
\hline TTR-PFOA & 149 & 1.1 & 0.60 & 1.88 & 1.68 \\
\hline TTR-TBBPA & 141 & 0.6 & 0.75 & 3.76 & 2.95 \\
\hline TTR-TBBPA_nW & 151 & 1.3 & 0.65 & 3.11 & 2.09 \\
\hline
\end{tabular}

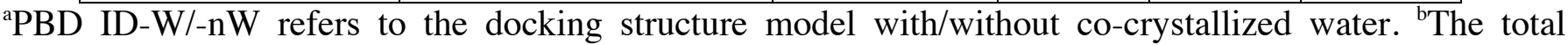
number of active TTR binders identified by the given single structure model; ${ }^{\text {cThe }}$ root-mean-squareddeviation between docking poses and co-crystalized poses of the co-ligands found in each X-ray structure. 
Table S6. Potential TTR binders (refined hits) proposed by the developed virtual screening protocol. For each compound, the protonation form with a more negative docking score (considering both binding affinity and tautomer ratio) was shown as below.

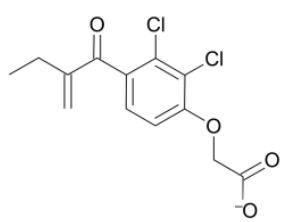

Compounds: Ethacrynic acid
CAS Nr.: $58-54-8$
DockingScores: -8.72
Electro: 5
Unpolar: 16
pKa1: 2.8
pKa2: $\mathrm{NA}$
Molecular charge: -1

Compounds: Cefdinir

CAS Nr.: 91832-40-5

DockingScores: -7.6

Electro: 5

Unpolar: 5

pKa1: 1.74

pKa2: 3.2

Molecular charge: -1

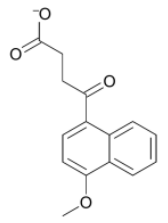

Compounds: Menbutone

CAS Nr.: 3562-99-0

DockingScores: -9.02

Electro: 4

Unpolar: 9

pKa1: 4.05

pKa2: 16.88

Molecular charge: -1

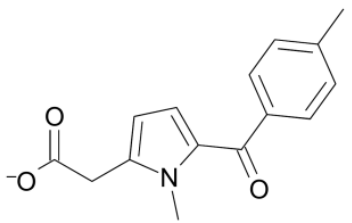

Compounds: Tolmetin sodium

CAS Nr.: 64490-92-2

DockingScores: -8.59

Electro: 4

Unpolar: 8

pKa1: 3.96

pKa2: NA

Molecular charge: -1
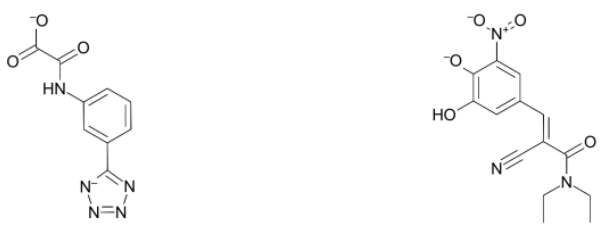

Compounds: Acitazanolast

CAS Nr.: 114607-46-4

DockingScores: -8.1

Electro: 5

Unpolar: 3

pKa1: 2.45

pKa2: 4.28

Molecular charge: -2

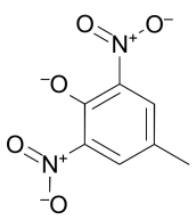

Compounds: Entacapone

CAS Nr: : 130929-57-6

DockingScores: -7.85

Electro: 5

Unpolar: 5

pKa1: 5.94

pKa2: 10.41

Molecular charge: -1

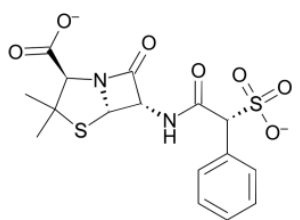

Compounds: 4-Methyl-2,6-dinitrophenol Compounds: Sulbenicillin disodium

CAS Nr.: 609-93-8

DockingScores: -6.55

Electro: 5

Unpolar: 2

pKa1: 4.57

pKa2: NA

Molecular charge: -1

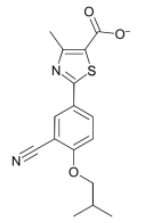

Compounds: Febuxostat

CAS Nr.: 144060-53-7

DockingScores: -8.98

Electro: 4

Unpolar: 9

pKa1: 3.59

pKa2: NA

Molecular charge: -1

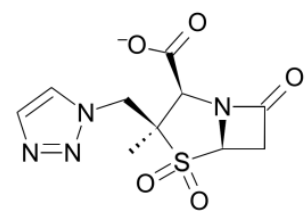

Compounds: Tazobactam sodium

CAS Nr.: 89785-84-2

DockingScores: -8.49

Electro: 4

Unpolar: 2

pKa1: 2.86

pKa2: 18.64

Molecular charge: -1

CAS Nr.: 28002-18-8

DockingScores: -9.83

Electro: 4

Unpolar: 14

pKa1: -1.09

pKa2: 3.13

Molecular charge: -2

Compounds: Trepibutone

CAS Nr.: 41826-92-0

DockingScores: -8.86

Electro: 4

Unpolar: 9

pKa1: 3.45

pKa2: 16.46

Molecular charge: -1<smiles>O=C([O-])c1cc(C(=O)[O-])c(C(=O)[O-])cc1C(=O)[O-]</smiles>

Compounds: Pyromellitic acid

CAS Nr: : 89-05-4

DockingScores: -8.38

Electro: 4

Unpolar: 4

pKa1: 1.97

pKa2: 2.93

Molecular charge: -4<smiles>CCOc1cc(OCC)c(C(=O)CCC(=O)O)cc1OCC</smiles>

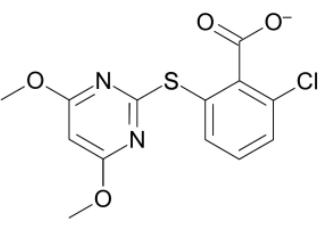

Compounds: Pyrithiobac-sodium

CAS Nr: : 123343-16-8

DockingScores: -7.71

Electro: 5

Unpolar: 5

pKa1: 2.21

pKa2: NA

Molecular charge: -1<smiles>CC1(C)S[C@@H]2[C@H](NC(=O)[C@H](C(=O)[O-])c3ccccc3)C(=O)N2[C@H]1C(=O)O</smiles>

Compounds: Carbenicillin disodium

CAS Nr: : 4800-94-6

DockingScores: -9.48

Electro: 4

Unpolar: 15

pKa1: 3.11

pKa2: 3.86

Molecular charge: -2<smiles>Nc1cc(S(=O)(=O)[O-])cc2cc(S(=O)(=O)[O-])cc(O)c12</smiles>

Compounds: 2,7-Naphthalenedisulfonic acid, 4-amino-5-hydroxy-, monosodium salt hydrate

CAS Nr.: 312693-54-2

DockingScores: -8.59

Electro: 4

Unpolar: 4

pKa1: -3.74

pKa2: -2.66

Molecular charge: -2

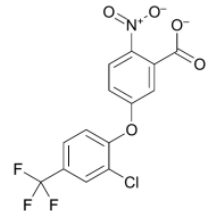

Compounds: Acifluorfen-sodium

CAS Nr. : 62476-59-9

DockingScores: -8.34

Electro: 4

Unpolar: 11

pKa1: 1.36

pKa2: NA

Molecular charge: -1 
<smiles>O=C([O-])c1cc2ccccc2nc1C(=O)[O-]</smiles>

Compounds: 2,3-Quinolinedicarboxylic acid

DockingScores: -8.32

Electro: 4

Unpolar: 7

pKa1: 0.41

pKa2: 4.04

Molecular charge: -2<smiles>O=C(O)c1ccc(C(=O)[O-])c(C(=O)[O-])c1</smiles>

CAS Nr.: 643-38-9<smiles>O=C([O-])c1cccc(C(=O)[O-])c1C(=O)[O-]</smiles>

Compo acid

CAS Nr.: 36362-97-7

DockingScores: -8.29

Electro: 4

Unpolar: 3

pKa1: 2.23

pKa2: 3.99

Molecular charge: -3<smiles>O=C(O)c1cc2ccccc2cc1O</smiles>

Compounds: 1,2,4-Benzenetricarboxylic Compounds: 3-Hydroxy-2-naphthoic acid

CAS Nr.: $528-44-9$

DockingScores: -8.17

Electro: 4

Unpolar: 3

pKa1: 2.52

pKa2: 3.88

Molecular charge: -3<smiles>CCN(CC)S(=O)(=O)c1ccc(C(=O)O)cc1</smiles>

Compounds: Ethebenecid

CAS Nr: : $1213-06-5$

DockingScores: -7.78

Electro: 4

Unpolar: 6

pKa1: 3.53

pKa2: NA

Molecular charge: -1<smiles>Cc1[nH+]cc(COP(=O)([O-])[O-])c(CN)c1[O-]</smiles>

Compounds: Pyridoxamine phosphate CAS Nr.: 529-96-4

DockingScores: -7.49

Electro: 4

Unpolar: 4

pKa1: 1.74

pKa2: 6.74

Molecular charge: -1 acid

CAS Nr: : 92-70-6

DockingScores: -8.17

Electro: 4

Unpolar: 5

pKa1: 2.69

pKa2: 12.83

Molecular charge: -1

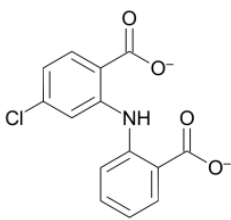

CAS Nr.: 64808-48-6

DockingScores: -7.75

Electro: 4

Unpolar: 8

pKa1: 3.51

pKa2: 4.11

Molecular charge: -2

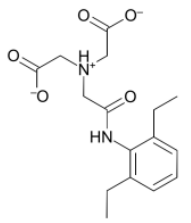

Compounds: Etifenin

CAS Nr.: 63245-28-3

DockingScores: -7.4

Electro: 4

Unpolar: 10

pKa1: 3.26

pKa2: 5.56

Molecular charge: -1
Compounds: Lobenzarit sodium

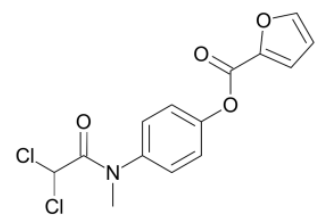

Compounds: Diloxanide furoate CAS Nr: : 3736-81-0

DockingScores: -8.29

Electro: 4

Unpolar: 12

pKa1: 13.09

pKa2: NA

Molecular charge: 0

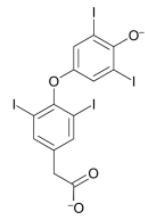

Compounds: 3,3',5,5'-

Tetraiodothyroacetic acid

CAS Nr: : 67-30-1

DockingScores: -8.14

Electro: 4

Unpolar: 4

pKa1: 2.25

pKa2: 7.43

Molecular charge: -2<smiles>CC(=O)NC(Cc1ccc(O)cc1)C(=O)[O-]</smiles>

Compounds: N-Acetyl-L-tyrosine CAS Nr: : 537-55-3

DockingScores: -7.66

Electro: 4

Unpolar: 4

pKa1: 3.67

pKa2: 9.5

Molecular charge: -1<smiles>O=C(O)CSCC(=O)N[C@H]1CCSC1=O</smiles>

Compounds: Erdosteine

CAS Nr: : 84611-23-4

DockingScores: -7.35

Electro: 4

Unpolar: 6

pKa1: 3.79

pKa2: 12.2

Molecular charge: -1

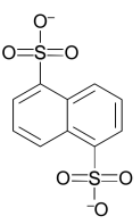

Compounds: 1,5-Naphthalenedisulfonic acid, disodium salt hydrate CAS Nr.: 207569-02-6

DockingScores: -8.2

Electro: 4

Unpolar: 4

pKa1: -2.72

pKa2: -1.93

Molecular charge: -2<smiles>O=S(=O)([O-])c1ccc2cc(O)ccc2c1</smiles>

Compounds: Sodium 6

hydroxynaphthalene-2-sulfonate

CAS Nr: : 135-76-2

DockingScores: -7.88

Electro: 4

Unpolar: 7

pKa1: -2.04

pKa2: 9.78

Molecular charge: -1<smiles>Cc1[nH+]cc(COP(=O)([O-])[O-])c(C=O)c1[O-]</smiles>

Compounds: Pyridoxal phosphate hydrate

CAS Nr.: 41468-25-1

DockingScores: -7.63

Electro: 4

Unpolar: 3

pKa1: 1.68

pKa2: 6.69

Molecular charge: -2<smiles>O=c1c(O)c(-c2ccc(O)c(O)c2)oc2cc(O)cc(O)c12</smiles>

Compounds: Quercetin dihydrate

CAS Nr.: 6151-25-3

DockingScores: -7.32

Electro: 4

Unpolar: 4

pKa1: 6.38

pKa2: 7.85

Molecular charge: 0 
<smiles>COC(=O)[C@H](N)CC1=CN=C2C=CC(O)=CC12</smiles>

Compounds: L-5-Hydroxytryptophan CAS Nr.: 4350-09-8

DockingScores: -7.32

Electro: 4

Unpolar: 4

pKa1: 2.15

pKa2: 9.8

Molecular charge: 0<smiles>O=C([O-])c1ccc(Cl)c(Cl)c1</smiles>

Compounds: 3,4-Dichlorobenzoic acid CAS Nr: : 51-44-5

DockingScores: -7.17

Electro: 4

Unpolar: 6

pKa1: 3.93

pKa2: NA

Molecular charge: -1<smiles>O=S(=O)([O-])c1cc(O)c(O)c(S(=O)(=O)[O-])c1</smiles>

Compounds: Tiron

CAS Nr: : 149-45-1

DockingScores: -6.92

Electro: 4

Unpolar: 2

pKa1: -3.46

pKa2: -2.66

Molecular charge: -2

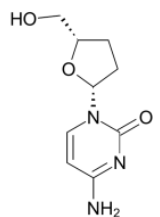

Compounds: 2', 3'-Dideoxycytidine

CAS Nr.: 7481-89-2

DockingScores: -6.62

Electro: 4

Unpolar: 4

pKa1: 14.67

pKa2: 19.22

Molecular charge: 0<smiles>COC(=O)CNC(=O)c1ccccc1</smiles><smiles>Cc1cc(Cl)ccc1OCC(=O)[O-]</smiles><smiles>O=C(O)c1c(Cl)ccc(Cl)c1Cl</smiles>

Compounds: Hippuric acid

CAS Nr.: 495-69-2

DockingScores: -7.32

Electro: 4

Unpolar: 3

pKa1: 3.59

pKa2: 15.09

Molecular charge: -1

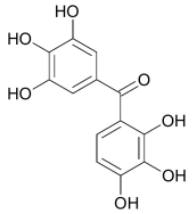

Compounds: Exifone

CAS Nr.: 52479-85-3

DockingScores: -7.02

Electro: 4

Unpolar: 5

pKa1: 7.08

pKa2: 7.7

Molecular charge: 0

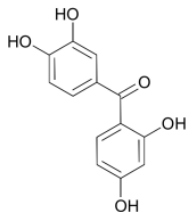

Compounds: 2,3',4,4'-

Tetrahydroxybenzophenone

CAS Nr.: 61445-50-9

DockingScores: -6.92

Electro: 4

Unpolar: 6

pKa1: 7.03

pKa2: 7.95

Molecular charge: 0

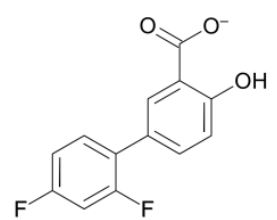

Compounds: Diflunisal

CAS Nr.: 22494-42-4

DockingScores: -9.23

Electro: 3

Unpolar: 4

pKa1: 2.69

pKa2: 12.68

Molecular charge: -1
Compounds: MCPA-sodium

CAS Nr.: 3653-48-3

DockingScores: -7.3

Electro: 4

Unpolar: 5

pKa1: 3.36

pKa2: NA

Molecular charge: -1

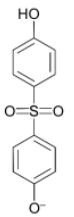

Compounds: 4,4'-Sulfonyldiphenol

CAS Nr.: 80-09-1

DockingScores: -6.97

Electro: 4

Unpolar: 7

pKa1: 7.42

pKa2: 8.03

Molecular charge: -1<smiles>O=C([O-])c1cc(/N=N/c2ccc(O)c(C(=O)[O-])c2)ccc1O</smiles>

Compounds: Olsalazine sodium CAS Nr: : 6054-98-4

DockingScores: -6.82

Electro: 4

Unpolar: 6

pKa1: 2.93

pKa2: 3.53

Molecular charge: -2

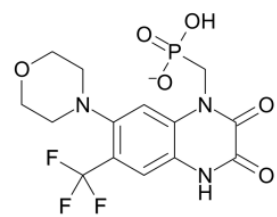

Compounds: Fanapanel

CAS Nr: 161605-73-8

DockingScores: -9.22

Electro: 3

Unpolar: 5

pKa1: 1.54

pKa2: 8.07

Molecular charge: -1
Compounds: 2,3,6-Trichlorobenzoic acid CAS Nr: : $50-31-7$

DockingScores: -7.28

Electro: 4

Unpolar: 4

pKa1: 1.9

pKa2: NA

Molecular charge: -1<smiles>O=C([O-])c1ccc(O)cc1O</smiles>

Compounds: 2,4-Dihydroxybenzoic acid CAS Nr.: 89-86-1

DockingScores: -6.96

Electro: 4

Unpolar: 2

pKa1: 3.1

pKa2: 9.8

Molecular charge: -1<smiles>O=C([O-])c1ccc(O)c(O)c1</smiles>

Compounds: 3,4-Dihydroxybenzoic acid CAS Nr.: 99-50-3

DockingScores: -6.76

Electro: 4

Unpolar: 2

pKa1: 4.16

pKa2: 9.4

Molecular charge: -1<smiles>O=S(=O)([O-])c1ccc2cc(O)c(S(=O)(=O)[O-])cc2c1</smiles>

Compounds: Disodium 3-

hydroxynaphthalene-2,7-disulfonate

CAS Nr.: 135-51-3

DockingScores: -8.99

Electro: 3

Unpolar: 8

pKa1: -2.89

pKa2: -2.11

Molecular charge: -2 
<smiles>C=N[C@H](C(=O)N[C@H]1C(=O)N2C(C(=O)O)C(C)(C)S[C@H]12)c1ccccc1</smiles><smiles>C[C@H](C(=O)[O-])c1ccc(C(=O)c2ccccc2)s1</smiles>

Compounds: Metampicillin sodium CAS Nr.: 6489-61-8

DockingScores: -8.93

Electro: 3

Unpolar: 14

pKa1: 3.24

pKa2: 11.85

Molecular charge: -1<smiles>CC(C)(c1cc(Br)c([O-])c(Br)c1)c1cc(Br)c(O)c(Br)c1</smiles>

Compounds: 3,3',5,5'-

Tetrabromobisphenol A

CAS Nr.: 79-94-7

DockingScores: -8.79

Electro: 3

Unpolar: 2

pKa1: 6.54

pKa2: 7.17

Molecular charge: -1

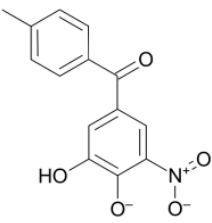

Compounds: Tolcapone

CAS Nr.: 134308-13-7

DockingScores: -8.53

Electro: 3

Unpolar: 8

pKa1: 5.44

pKa2: 10.31

Molecular charge: -1

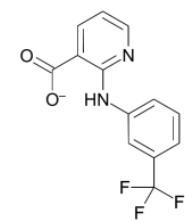

Compounds: Niflumic acid

CAS Nr.: 4394-00-7

DockingScores: -8.47

Electro: 3

Unpolar: 4

pKa1: 1.88

pKa2: 16.44

Molecular charge: -1

Compounds: Tiaprofenic acid

CAS Nr.: 33005-95-7

DockingScores: -8.91

Electro: 3

Unpolar: 13

pKa1: 4.03

pKa2: NA

Molecular charge: -1

Compounds: Penicillin G potassium

CAS Nr.: 113-98-4

DockingScores: -8.74

Electro: 3

Unpolar: 11

pKa1: 3.53

pKa2: 12.12

Compounds: N2-(4-Nitrophenyl)-L-

glutamine hydrochloride

CAS Nr.: 67953-08-6

DockingScores: -8.53

Electro: 3

Unpolar: 4

pKa1: 3.43

pKa2: 15.48

Molecular charge: -1

Compounds: Penicillin VK

CAS Nr.: 132-98-9

DockingScores: -8.46

Electro: 3

Unpolar: 12

pKa1: 3.39

pKa2: 11.74

Molecular charge: -1
Molecular charge: -1<smiles>CC1(C)SC2[C@H](N)C(=O)N2[C@H]1C(=O)[O-]</smiles><smiles>NC(=O)CC[C@H](Nc1ccc([N+](=O)[O-])cc1)C(=O)[O-]</smiles><smiles>CC1(C)SC2C(NC(=O)COc3ccccc3)C(=O)N2[C@H]1C(=O)O</smiles><smiles>O=C([O-])c1cc(O)c2c(c1)C(=O)c1cccc(O)c1C2=O</smiles>

Compounds: Rhein

CAS Nr.: 478-43-3

DockingScores: -8.89

Electro: 3

Unpolar: 8

pKa1: 3.4

pKa2: 7.89

Molecular charge: -1

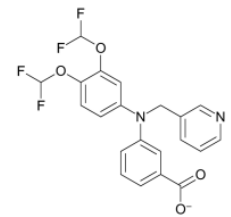

Compounds: $3-\{[3,4-$

Bis(difluoromethoxy)phenyl](pyridin-3ylmethyl)amino\}benzoic acid

CAS Nr.: 460081-99-6

DockingScores: -8.73

Electro: 3

Unpolar: 8

pKa1: 4.47

pKa2: NA

Molecular charge: -1<smiles>O=C(O)COc1ccc2ccccc2c1</smiles>

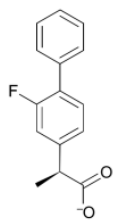

Compounds: Flurbiprofen

CAS Nr: : 5104-49-4

DockingScores: -8.83

Electro: 3

Unpolar: 11

pKa1: 4.42

pKa2: NA

Molecular charge: -1<smiles>O=C(O)C1=NN(c2ccc(S(=O)(=O)[O-])cc2)C(=O)C1</smiles>

Compounds: $1 \mathrm{H}-$ Pyrazole-3-carboxylic acid, 4,5-dihydro-5-oxo-1-(4-

sulfophenyl)-

CAS Nr.: 118-47-8

DockingScores: -8.62

Electro: 3

Unpolar: 4

pKa1: -2.43

pKa2: 2.04

Molecular charge: -2<smiles>CC(C(=O)O)c1ccc(C[C@H]2CCCC2=O)cc1</smiles>

Compounds: 2-Naphthoxyacetic acid

CAS Nr.: 120-23-0

DockingScores: -8.5

Electro: 3

Unpolar: 9

pKa1: 4

pKa2: NA

Molecular charge: -1

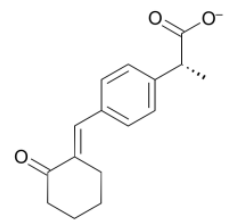

Compounds: Pelubiprofen

CAS Nr.: 69956-77-0

DockingScores: -8.32

Electro: 3

Unpolar: 10

pKa1: 4.11

pKa2: NA

Molecular charge: -1
Compounds: Loxoprofen

CAS Nr.: 68767-14-6

DockingScores: -8.5

Electro: 3

Unpolar: 11

pKa1: 4.19

pKa2: NA

Molecular charge: -1

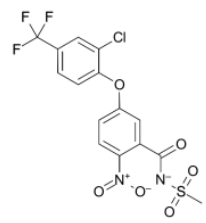

Compounds: Fomesafen

CAS Nr.: 72178-02-0

DockingScores: -8.28

Electro: 3

Unpolar: 10

pKa1: 3.93

pKa2: NA

Molecular charge: -1 
<smiles>O=C([O-])CC(NC(=O)OCc1ccccc1)C(=O)[O-]</smiles>

Compounds: N-[(Benzyloxy)carbonyl]-Laspartic acid

CAS Nr.: 1152-61-0

DockingScores: -8.24

Electro: 3

Unpolar: 10

pKa1: 3.47

pKa2: 5.22

Molecular charge: -2<smiles>O=C(O)c1ccc2cc(O)ccc2c1</smiles>

Compounds: 6-Hydroxy-2-naphthoic acid

CAS Nr: $16712-64-4$

DockingScores: -8.09

Electro: 3

Unpolar: 5

pKa1: 3.99

pKa2: 9.79

Molecular charge: -1<smiles>Cc1cccc(C)c1NC(=O)c1ccccc1C(=O)[O-]</smiles>

Compounds: Ftaxilide

CAS Nr.: 19368-18-4

DockingScores: -8.04

Electro: 3

Unpolar: 12

pKa1: 2.91

pKa2: 14.39

Molecular charge: -1<smiles>O=C([O-])c1ccc(S(=O)(=O)[O-])cc1C(=O)[O-]</smiles>

Compounds: 1,2-Benzenedicarboxylic acid, 4-sulfo-

CAS Nr.: 89-08-7

DockingScores: -7.91

Electro: 3

Unpolar: 3

pKa1: -2.85

pKa2: 2.78

Molecular charge: -3

Compounds: 2-(2,4,5-<smiles>CN1C2=CC(=O)/C(=N\NC(N)=O)C=C2C[C@H]1S(=O)(=O)[O-]</smiles>

Compounds: Carbazochrome sodium sulfonate

CAS Nr: : 51460-26-5

DockingScores: -8.17

Electro: 3

Unpolar: 3

pKa1: -1.29

pKa2: 11.64

Molecular charge: -1<smiles>COC(=O)CCCOc1ccc(Cl)cc1Cl</smiles>

Compounds: 4-(2,4-

Dichlorophenoxy)butyric acid

CAS Nr.: 94-82-6

DockingScores: -8.07

Electro: 3

Unpolar: 7

pKa1: 3.58

pKa2: NA

Molecular charge: -1

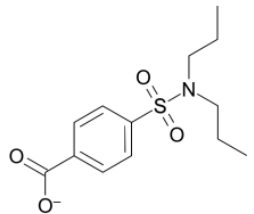

Compounds: Probenecid

CAS Nr. : 57-66-9

Electro: 3

Unpolar: 9

pKa1: 3.53

pKa2: NA

Molecular charge: -1

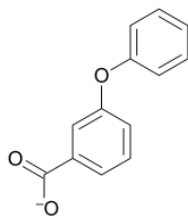

Compounds: 3-Phenoxybenzoic acid

CAS Nr: : 3739-38-6

DockingScores: -8.07

Electro: 3

Unpolar: 8

pKa1: 3.82

pKa2: NA

Molecular charge: -1<smiles>O=c1[n-]c(=O)n(Cl)c(=O)n1Cl</smiles>

Compounds: 2-(4-Aminophenyl)-6methylbenzothiazole sulfonic acid

CAS Nr.: 130-17-6

DockingScores: -8.03

Electro: 3

Unpolar: 9

pKa1: -3.17

pKa2: NA

Molecular charge: -1<smiles>C[C@H](Oc1cc(Cl)c(Cl)cc1Cl)C(=O)[O-]</smiles>

Trichlorophenoxy)propionic acid

CAS Nr.: 93-72-

DockingScores: -7.91

Electro: 3

Unpolar: 7

pKa1: 2.7

pKa2: NA

Molecular charge: -1

Compounds: Sodium dichloro-s-

riazinetrione dihydrate

CAS Nr.: 51580-86-0

DockingScores: -7.97

Electro: 3

Unpolar: 2

pKa1: 5.77

pKa2: NA

Molecular charge: -1<smiles>CCc1cnc(C(=O)[O-])c(C(=O)[O-])c1</smiles>

Compounds: 5-Ethyl-2,3-

pyridinedicarboxylic acid

CAS Nr.: 102268-15-5

DockingScores: -7.9

Electro: 3

Unpolar: 2

pKa1: 0.46

pKa2: 4.13

Molecular charge: -2
DockingScores: -8.17

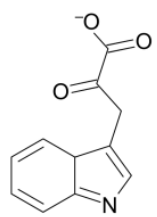

Compounds: Indole-3-pyruvic acid

CAS Nr.: 392-12-1

DockingScores: -8.12

Electro: 3

Unpolar: 4

pKa1: 3.49

pKa2: 15.04

Molecular charge: -1<smiles>COC(=O)CCCC[C@H]1SC[C@@H]2NC(=O)N[C@@H]21</smiles>

Compounds: Biotin

CAS Nr. : 58-85-5

DockingScores: -8.05

Electro: 3

Unpolar: 4

pKa1: 4.4

pKa2: 13.55

Molecular charge: -1

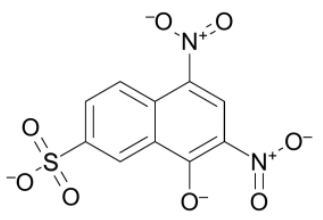

Compounds: 8-Hydroxy-5,7-dinitro-2naphthalenesulfonic acid

CAS Nr.: 483-84-1

DockingScores: -7.96

Electro: 3

Unpolar: 3

pKa1: -2.7

pKa2: 4.02

Molecular charge: -2<smiles>COC(=O)c1ccc(-n2nc(C)cc2O)cc1</smiles>

Compounds: 4-(3-Methyl-5-oxo-4,5dihydro-1H-pyrazol-1-yl)benzoic acid

CAS Nr: $60875-16-3$

DockingScores: -7.87

Electro: 3

Unpolar: 6

pKa1: 4.35

pKa2: 5.95

Molecular charge: -1 


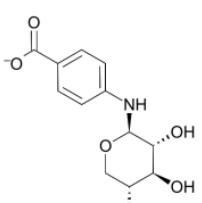

OH

Compounds: Benaxibine

CAS Nr.: 27661-27-4

DockingScores: -7.85

Electro: 3

Unpolar: 3

pKa1: 4.69

pKa2: 12.33

Molecular charge: -1<smiles>O=C1C=C2CC[C@H]3C4CC[C@](O)(C(=O)COP(=O)([O-])[O-])C4C[C@H](O)[C@@H]3C2CC1</smiles>

Compounds: Hydrocortisone sodium phosphate

CAS Nr.: 6000-74-4

DockingScores: -7.82

Electro: 3

Unpolar: 19

pKa1: 1.18

pKa2: 6.21

Molecular charge: -2<smiles>O=C([O-])Cc1ccc(Cl)cc1Cl</smiles>

Compounds: 2,4-Dichlorophenylacetic acid

CAS Nr.: 19719-28-9

DockingScores: -7.85

Electro: 3

Unpolar: 6

pKa1: 3.32

pKa2: NA

Molecular charge: -1

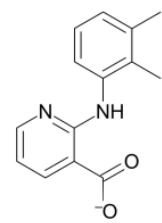

Compounds: Nixylic acid

CAS Nr.: 4394-05-2

DockingScores: -7.82

Electro: 3

Unpolar: 6

pKa1: 1.89

pKa2: 16.68

Molecular charge: -1<smiles>COC(=O)C1=C(OC)CS[C@@H]2[C@H](NC(=O)[C@@H](N)C3=CCC=CC3)C(=O)N12</smiles>

Compounds: Cefroxadine

CAS Nr.: 51762-05-1

DockingScores: -7.74

Electro: 3

Unpolar: 13

pKa1: 3.28

pKa2: 11.9

Molecular charge: -1<smiles>COC(=O)COc1cc(Cl)c(Cl)cc1Cl</smiles>

Compounds: 2,4,5-

Trichlorophenoxyacetic acid

CAS Nr.: 93-76-5

DockingScores: -7.69

Electro: 3

Unpolar: 7

pKa1: 2.56

pKa2: NA

Molecular charge: -1<smiles>CC(C)(Oc1ccc(Cl)cc1)C(=O)[O-]</smiles>

Compounds: Clofibric acid

CAS Nr.: 882-09-7

DockingScores: -7.74

Electro: 3

Unpolar: 7

pKa1: 3.37

pKa2: NA

Molecular charge: -1

Compounds: Ampicillin trihydrate

CAS Nr. : 7177-48-2

DockingScores: -7.68

Electro: 3

Unpolar: 11

pKa1: 3.24

pKa2: 11.97

Molecular charge: 0

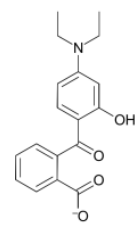

Compounds: 2-[4-(Diethylamino)-2hydroxybenzoyl]benzoic acid

CAS Nr.: 5809-23-4

DockingScores: -7.83

Electro: 3

Unpolar: 7

pKa1: 3.72

pKa2: 7.32

Molecular charge: -1<smiles>CC(C)(c1cc(Cl)c([O-])c(Cl)c1)c1cc(Cl)c(O)c(Cl)c1</smiles>

Compounds: 3,3 ', 5, 5'-

Tetrachlorobisphenol A

CAS Nr.: 79-95-8

DockingScores: -7.79

Electro: 3

Unpolar: 2

pKa1: 6.27

pKa2: 6.88

Molecular charge: -1<smiles>Cc1ccc(Cl)c(Nc2ccccc2C(=O)O)c1Cl</smiles>

Compounds: Meclofenamate sodium

CAS Nr.: 6385-02-0

DockingScores: -7.73

Electro: 3

Unpolar: 9

pKa1: 3.79

pKa2: 17.05

Molecular charge: -1<smiles>CC1(C)S[C@@H]2[C@H](NC(=O)[C@@H]([NH3+])c3ccccc3)C(=O)N2C1C(=O)[O-]</smiles><smiles>[NH3+]C(C(=O)N[C@@H]1C(=O)N2C(C(=O)[O-])=C(Cl)CC[C@H]12)c1ccccc1</smiles>

Compounds: Loracarbef hydrate<smiles>C[C@H](Oc1cc(Cl)ccc1Cl)C(=O)[O-]</smiles>

Compounds: alpha-(2,5-

Dichlorophenoxy)propionic acid

CAS Nr.: 6965-71-5

DockingScores: -7.83

Electro: 3

Unpolar: 6

pKa1: 2.95

Molecular charge: -1<smiles>COC(=O)[C@H](C)Oc1cccc(Cl)c1</smiles>

Compounds: Cloprop

CAS Nr.: 101-10-0

DockingScores: -7.76

Electro: 3

Unpolar: 6

pKa1: 3.27

pKa2: NA

Molecular charge: -1<smiles>O=C(O[C@H](C(=O)[O-])[C@H](OC(=O)c1ccccc1)C(=O)O)c1ccccc1</smiles>

Compounds: Dibenzoyl-L-tartaric acid

CAS Nr. : 2743-38-6

DockingScores: -7.71

Electro: 3

Unpolar: 16

pKa1: 2.77

pKa2: 4.74

Molecular charge: -2

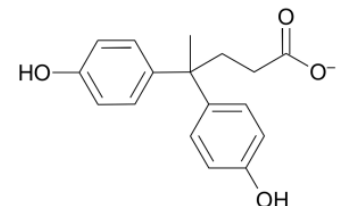

Compounds: Diphenolic acid

CAS Nr. : 126-00-1

DockingScores: -7.62

Electro: 3

Unpolar: 7

pKa1: 4.22

pKa2: 9.19

Molecular charge: -1 
<smiles>O=C([O-])C1CCC(C(=O)O)CC1</smiles>

Compounds: 1,4

Cyclohexanedicarboxylic acid

CAS Nr.: 1076-97-7

DockingScores: -7.6

Electro: 3

Unpolar: 3

pKa1: 3.9

pKa2: 4.65

Molecular charge: -2<smiles>O=C([O-])COc1ccc(Cl)cc1Cl</smiles>

Compounds: 2,4-D, Dimethylamine salt CAS Nr.: 2008-39-1

DockingScores: -7.57

Electro: 3

Unpolar: 5

pKa1: 2.81

pKa2: NA

Molecular charge: -1<smiles>O=C(O)[C@H](O)c1ccccc1</smiles>

Compounds: D-Mandelic acid

CAS Nr.: 611-71-2

DockingScores: -7.54

Electro: 3

Unpolar: 3

pKa1: 3.75

pKa2: 13.57

Molecular charge: -1<smiles>O=C([O-])CNC(=O)c1ccc([N+](=O)[O-])cc1</smiles>

Compounds: 4-Nitrohippuric acid CAS Nr.: 2645-07-0

DockingScores: -7.5

Electro: 3

Unpolar: 2

pKa1: 2.72

pKa2: 13.78

Molecular charge: -1<smiles>CC1=C(C(=O)[O-])N2C(=O)[C@@H](NC(=O)[C@H](N)C3=CCC=CC3)[C@H]2SC1</smiles><smiles>Cc1cc(Cl)ccc1OCCCC(=O)[O-]</smiles><smiles>COC(=O)/C=C/[C@@H](O)c1ccc(Cl)cc1</smiles>

Compounds: Cephradine

CAS Nr.: 38821-53-3

DockingScores: -7.6

Electro: 3

Unpolar: 12

pKa1: 3.46

pKa2: 11.99

Molecular charge: -1

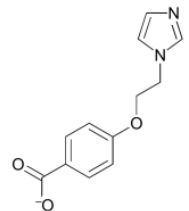

Compounds: Dazoxiben

CAS Nr.: 78218-09-4

DockingScores: -7.57

Electro: 3

Unpolar: 2

pKa1: 4.36

pKa2: NA

Molecular charge: -1<smiles>Cc1c(Cl)cccc1Nc1ncccc1C(=O)O</smiles>

Compounds: Clonixin

CAS Nr.: 17737-65-4

DockingScores: -7.54

Electro: 3

Unpolar: 5

pKa1: 1.88

pKa2: 16.22

Molecular charge: -1<smiles>O=C(O[Na])c1c(Cl)cccc1Cl</smiles>

Compounds: 2,6-Dichlorobenzoic acid CAS Nr.: 50-30-6

DockingScores: -7.5

Electro: 3

Unpolar: 4

pKa1: 2.06

pKa2: NA

Molecular charge: -1
Compounds: 4-(2-Methyl-4chlorophenoxy)butyric acid CAS Nr.: 94-81-5

DockingScores: -7.59

Electro: 3

Unpolar: 9

pKa1: 4.06

pKa2: NA

Molecular charge: -1<smiles>CC(C)(C)c1ccc(C(=O)[O-])cc1</smiles>

Compounds: 4-tert-Butylbenzoic acid CAS Nr.: 98-73-7

DockingScores: -7.56

Electro: 3

Unpolar: 6

pKa1: 4.22

pKa2: NA

Molecular charge: -1<smiles>Cc1cccc([N+](=O)[O-])c1C(=O)[O-]</smiles>

Compounds: 2-Methyl-6-nitrobenzoic acid

CAS Nr: : 13506-76-8

DockingScores: -7.54

Electro: 3

Unpolar: 4

pKa1: 1.58

pKa2: NA

Molecular charge: -1<smiles>COC(=O)COc1ccc(Cl)cc1</smiles>

Compounds: 4-Chlorophenoxyacetic acid

CAS Nr.: 122-88-3

DockingScores: -7.48

Electro: 3

Unpolar: 4

pKa1: 3.14

pKa2: NA

Molecular charge: -1
Compounds: sodium (2E)-4-(4-

chlorophenyl)-4-hydroxybut-2-enoate hydrate

CAS Nr.: 1049737-99-6

DockingScores: -7.58

Electro: 3

Unpolar: 3

pKa1: 4.07

pKa2: 14.07

Molecular charge: -1<smiles>O=C([O-])c1ccccc1C(=O)[O-]</smiles>

Compounds: Monopotassium phthalate CAS Nr: : 877-24-7

DockingScores: -7.55

Electro: 3

Unpolar: 3

pKa1: 2.94

pKa2: 5.49

Molecular charge: -2<smiles>COC(=O)c1cccc(C)c1[N+](=O)[O-]</smiles>

Compounds: 3-Methyl-2-nitrobenzoic acid

CAS Nr: : 5437-38-7

DockingScores: -7.53

Electro: 3

Unpolar: 3

pKa1: 1.69

pKa2: NA

Molecular charge: -1

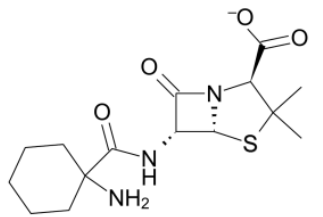

Compounds: Cyclacillin

CAS Nr.: 3485-14-1

DockingScores: -7.47

Electro: 3

Unpolar: 16

pKa1: 3.3

pKa2: 12.08

Molecular charge: -1 
<smiles>O=C(O)COc1nc(Cl)c(Cl)cc1Cl</smiles>

Compounds: Triclopyr CAS Nr.: 55335-06-3 DockingScores: -7.44 Electro: 3

Unpolar: 6

pKa1: 2.28

pKa2: NA

Molecular charge: -1

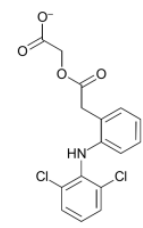

Compounds: Aceclofenac

CAS Nr.: 89796-99-6

DockingScores: -7.41

Electro: 3

Unpolar: 13

pKa1: 3.44

pKa2: 16.4

Molecular charge: -1<smiles>Cc1c(C(=O)O)cccc1[N+](=O)[O-]</smiles>

Compounds: 2-Methyl-3-nitrobenzoic

acid CAS Nr.: 1975-50-4

DockingScores: -7.39

Electro: 3

Unpolar: 3

pKa1: 3.43

pKa2: NA

Molecular charge: -1<smiles>O=c1c(-c2ccc(O)cc2)coc2cc(O)cc(O)c12</smiles>

Compounds: Genistein

CAS Nr.: 446-72-0

DockingScores: -7.31

Electro: 3

Unpolar: 10

pKa1: 6.55

pKa2: 8.09

Molecular charge: 0

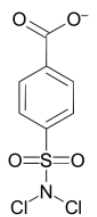<smiles>Cc1cc(Cl)ccc1O[C@@H](C)C(=O)[O-]</smiles>

Compounds: Halazone

CAS Nr.: 80-13-7

DockingScores: -7.44

Electro: 3

Unpolar: 6

pKa1: 3.47

pKa2: NA

Molecular charge: -1<smiles>COc1c(Cl)ccc(Cl)c1C(=O)[O-]</smiles>

Compounds: Dicamb

CAS Nr.: 1918-00-9

DockingScores: -7.4

Electro: 3

Unpolar: 4

pKa1: 2.54

pKa2: NA

Molecular charge: -1<smiles>CC1=C(C(=O)[O-])N2C(=O)[C@@H](NC(=O)C([NH3+])c3ccccc3)[C@H]2SC1</smiles>

Compounds: Cephalexin

CAS Nr.: 15686-71-2

DockingScores: -7.38

Electro: 3

Unpolar: 14

pKa1: 3.45

pKa2: 11.91

Molecular charge: 0<smiles>CC(=O)Oc1cccc(C(=O)O)c1OC(C)=O</smiles>

Compounds: Dipyrocetyl

CAS Nr.: 486-79-3

DockingScores: -7.31

Electro: 3

Unpolar: 4

pKa1: 3.16

pKa2: NA

Molecular charge: -1

Compounds: Mecoprop

CAS Nr: : 93-65-2

DockingScores: -7.44

Electro: 3

Unpolar: 4

pKa1: 3.47

pKa2: NA

Molecular charge: -1

Compounds: Amoxicillin

CAS Nr.: 26787-78-0

DockingScores: -7.39

Electro: 3

Unpolar: 9

pKa1: 3.23

pKa2: 9.48

Molecular charge: 0<smiles>O=C(O)c1cc(=O)c2ccccc2o1</smiles>

Compounds: Chromocarb

CAS Nr: : 4940-39-0

DockingScores: -7.33

Electro: 3

Unpolar: 2

pKa1: 2.25

pKa2: 15.64

Molecular charge: -1<smiles>O=C(O)c1cc(C(=O)O)cc(S(=O)(=O)[O-])c1</smiles>

Compounds: Monosodium 5sulfoisophthalate

CAS Nr.: 6362-79-4

DockingScores: -7.3

Electro: 3

Unpolar: 3

pKa1: -2.88

pKa2: 3.4

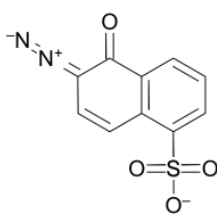

Compounds: 1-Naphthalenesulfonic acid, 6-diazo-5,6-dihydro-5-oxo, sodium salt

CAS Nr: : 2657-00-3

DockingScores: -7.43

Electro: 3

Unpolar: 5

pKa1: -2.44

pKa2: 8.73

Molecular charge: -1<smiles>CC1(C)S[C@@H]2[C@H](NC(=O)[C@H]([NH3+])c3ccc(O)cc3)C(=O)N2C1C(=O)O</smiles><smiles>O=C(O)CNC(=O)c1ccccc1I</smiles>

Molecular charge: -3
Compounds: 2-Iodohippuric acid

CAS Nr: : 147-58-0

DockingScores: -7.39

Electro: 3

Unpolar: 5

pKa1: 2.65

pKa2: 13.61

Molecular charge: -1<smiles>COC(=O)C(=O)Cc1ccccc1</smiles>

Compounds: Phenylpyruvic acid

CAS Nr.: 156-06-9

DockingScores: -7.33

Electro: 3

Unpolar: 4

pKa1: 3.33

pKa2: 16.

Molecular charge: -1<smiles>CC(C)c1ccc(S(=O)(=O)[O-])cc1</smiles>

Compounds: 4-(Propan-2-

yl)benzenesulfonic acid

CAS Nr: : 16066-35-6

DockingScores: -7.3

Electro: 3

Unpolar: 5

pKa1: -1.87

pKa2: NA

Molecular charge: -1 


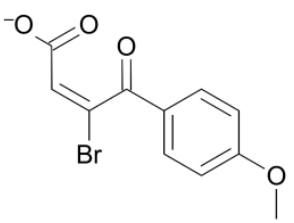

Compounds: Cytembena

CAS Nr.: 21739-91-3

DockingScores: -7.3

Electro: 3

Unpolar: 4

pKa1: 1.74

pKa2: NA

Molecular charge: -1

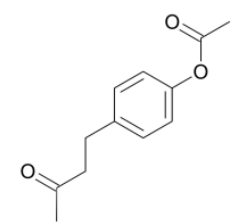

Compounds: 4-(4-(Acetyloxy)phenyl)-2- Compounds: Cefaclor

\section{butanone}

CAS Nr.: 3572-06-3

DockingScores: -7.27

Electro: 3

Unpolar: 8

pKa1: 19.59

pKa2: NA

Molecular charge: 0<smiles>NS(=O)(=O)c1ccc(C(=O)O)cc1</smiles>

Compounds: Carzenide

CAS Nr.: 138-41-0

DockingScores: -7.23

Electro: 3

Unpolar: 3

pKa1: 3.54

pKa2: 10.4

Molecular charge: -1<smiles>Cc1cc(C(=O)[O-])ccc1[N+](=O)[O-]</smiles>

Compounds: 3-Methyl-4-nitrobenzoic acid

CAS Nr.: 3113-71-1

DockingScores: -7.21

Electro: 3

Unpolar: 5

pKa1: 3.16

pKa2: NA

Molecular charge: -1

CAS Nr.: 69377-81-7

DockingScores: -7.29

Electro: 3

Unpolar: 4

pKa1: 2.41

pKa2: NA

Molecular charge: -1

CAS Nr.: 619-65-8

DockingScores: -7.23

Electro: 3

Unpolar: 4

pKa1: 3.54

pKa2: NA

Molecular charge: -1

methylbenzoic acid

CAS Nr. : 83-40-9

DockingScores: -7.2

Electro: 3

Unpolar: 3

pKa1: 2.8

pKa2: 13.89

Molecular charge: -1

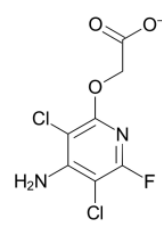

Compounds: Fluroxypyr<smiles>O=C([O-])c1ccc(Cl)cc1Cl</smiles><smiles>Cc1ccc(C(=O)O)cc1[N+](=O)[O-]</smiles><smiles>[NH3+]C(C(=O)N[C@@H]1C(=O)N2C(C(=O)[O-])=C(Cl)CS[C@H]12)c1ccccc1</smiles>

Compounds: Benzoic acid, 4-cyano-<smiles>Cc1cccc(C(=O)[O-])c1O</smiles>

Compounds: 2-Hydroxy-3-
Compounds: 2, 4-Dichlorobenzoic acid CAS Nr: : 50-84-0

DockingScores: -7.29

Electro: 3

Unpolar: 4

pKa1: 3.07

pKa2: NA

Molecular charge: -1<smiles>O=P([O-])(Oc1ccccc1)Oc1ccccc1</smiles>

Compounds: Diphenyl phosphate

CAS Nr.: 838-85-7

DockingScores: -7.25

Electro: 3

Unpolar: 12

pKa1: 0.87

pKa2: NA

Molecular charge: -1

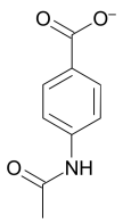

Compounds: 4-Methyl-3-nitrobenzoic acid

CAS Nr. : 96-98-0

DockingScores: -7.27

Electro: 3

Unpolar: 4

pKa1: 3.74

pKa2: NA

Molecular charge: -1<smiles>O=Cc1ccc(C(=O)O)cc1</smiles>

Compounds: 4-Carboxybenzaldehyde CAS Nr. : 619-66-9

DockingScores: -7.25

Electro: 3

Unpolar: 3

pKa1: 3.8

pKa2: NA

Molecular charge: -1

Compounds: 4-Acetamidobenzoic acid CAS Nr.: 556-08-1

DockingScores: -7.22

Electro: 3

Unpolar: 2

pKa1: 4.16

pKa2: 14.72

Molecular charge: -1<smiles>O=C(O)c1ccc([N+](=O)[O-])cc1</smiles>

Compounds: 4-Nitrobenzoic acid CAS Nr. : 62-23-7

DockingScores: -7.2

Electro: 3

Unpolar: 3

pKa1: 3.16

pKa2: NA

Molecular charge: -1<smiles>O=C([O-])c1cccc(Cl)c1Cl</smiles>

Compounds: 2,3-Dichlorobenzoic acid CAS Nr.: 50-45-3

DockingScores: -7.21

Electro: 3

Unpolar: 4

pKa1: 2.92

pKa2: NA

Molecular charge: -1<smiles>COC(=O)c1ccc(C(=O)[O-])cc1</smiles>

Compounds: 1,4-Benzenedicarboxylic acid, monomethyl ester

CAS Nr.: 1679-64-7

DockingScores: -7.19

Electro: 3

Unpolar: 3

pKa1: 3.62

pKa2: NA

Molecular charge: -1 
<smiles>O=C(NNCS(=O)(=O)[O-])c1ccncc1</smiles><smiles>Nc1cc([N+](=O)[O-])ccc1C(=O)[O-]</smiles>

Compounds: Isoniazid sodium methanesulfonate CAS Nr.: 3804-89-5 DockingScores: -7.18

Electro: 3

Unpolar: 3

pKa1: -1.53

pKa2: 13.63

Molecular charge: -1<smiles>O=C([O-])c1cc(Cl)ccc1Cl</smiles>

Compounds: 2,5-Dichlorobenzoic acid CAS Nr.: $50-79-3$

DockingScores: -7.17

Electro: 3

Unpolar: 4

pKa1: 2.88

pKa2: NA

Molecular charge: -1<smiles>O=C(O)Cc1c(Cl)ccc(Cl)c1Cl</smiles>

Compounds: Chlorfenac

CAS Nr: : 85-34-7

DockingScores: -7.09

Electro: 3

Unpolar: 8

pKa1: 3

pKa2: NA

Molecular charge: -1<smiles>Nc1cc(C(=O)[O-])ccc1Cl</smiles>

Compounds: 3-Amino-4-chlorobenzoic acid

CAS Nr.: 2840-28-0

DockingScores: -7.06

Electro: 3

Unpolar: 4

pKa1: 4.77

pKa2: NA

Molecular charge: -1

DockingScores: -7.16

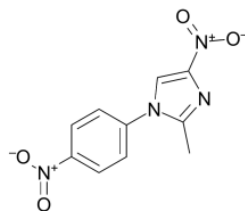

篮

Compounds: 4-Nitroanthranilic acid CAS Nr.: 619-17-0

DockingScores: -7.18

Electro: 3

Unpolar: 2

pKa1: 3.79

pKa2: 16.41

Molecular charge: -1

Compounds: Nitrefazole

CAS Nr: : 21721-92-6

DockingScores: -7.18

Electro: 3

Unpolar: 5

pKa1: -0.3

pKa2: NA

Molecular charge: 0<smiles>[NH3+]C[C@H](CC(=O)[O-])c1ccc(Cl)cc1</smiles>

Compounds: Baclofen

CAS Nr.: 1134-47-0

Electro: 3

Unpolar: 5

pKa1: 3.89

pKa2: NA

Molecular charge: 0

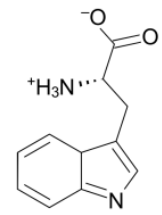

Compounds: I-Tryptophan

CAS Nr.: 73-22-3

DockingScores: -7.08

Electro: 3

Unpolar: 5

pKa1: 2.54

pKa2: 16.16

Molecular charge: 0

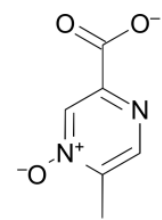

Compounds: Acipimox

CAS Nr.: 51037-30-0

DockingScores: -7.0

Electro: 3

Unpolar: 2

pKa1: -0.1

pKa2: NA

Molecular charge: -1<smiles>Nc1ccc(C(=O)NCC(=O)O)cc1</smiles>

Compounds: Aminohippuric acid

CAS Nr.: 61-78-9

DockingScores: -7.15

Electro: 3

Unpolar: 2

pKa1: 2.7

pKa2: 15.85

Molecular charge: -1

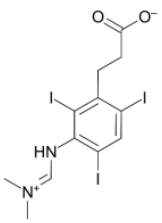

Compounds: Ipodate sodium

CAS Nr: : 1221-56-3

DockingScores: -7.06

Electro: 3

Unpolar: 7

pKa1: 2.48

pKa2: 10.81

Molecular charge: 0

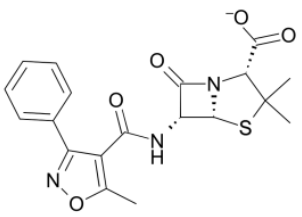

Compounds: Oxacillin sodium salt CAS Nr.: 1173-88-2

DockingScores: -6.99

Electro: 3

Unpolar: 8

pKa1: 3.75

pKa2: 13.65

Molecular charge: -1

\author{
Compounds: Benzadox \\ CAS Nr.: 5251-93-4 \\ DockingScores: -7.17 \\ Electro: 3 \\ Unpolar: 4 \\ pKa1: 3.41 \\ pKa2: 12.64 \\ Molecular charge: -1
}<smiles>O=C([O-])c1ccccc1S</smiles>

Compounds: Thiosalicylic acid

CAS Nr.: 147-93-3

DockingScores: -7.09

Electro: 3

Unpolar: 2

pKa1: 3.34

pKa2: 6.19

Molecular charge: -1<smiles>O=[N+]([O-])c1ccc(S(=O)(=O)[O-])c([N+](=O)[O-])c1</smiles>

Compounds: 2,4-dinitro Benzenesulfonic acid dihydrate

CAS Nr.: 89-02-1

DockingScores: -7.06

Electro: 3

Unpolar: 3

pKa1: -3.26

pKa2: 19.04

Molecular charge: -1<smiles>O=C([O-])c1nc(Cl)ccc1Cl</smiles>

Compounds: Clopyralid-olamine

CAS Nr.: 57754-85-5

DockingScores: -6.99

Electro: 3

Unpolar: 3

pKa1: 3.15

pKa2: NA

Molecular charge: -1 
<smiles>CC[C@@H](Cc1c(I)cc(I)c(N)c1I)C(=O)O</smiles>

Compounds: Iopanoic acid CAS Nr. : 96-83-3

DockingScores: -6.97

Electro: 3

Unpolar: 9

pKa1: 2.85

pKa2: 19.25

Molecular charge: -1<smiles>O=C([O-])c1cccc([N+](=O)[O-])c1</smiles>

Compounds: Sodium 3-nitrobenzoate CAS Nr.: 827-95-2

DockingScores: -6.92

Electro: 3

Unpolar: 2

pKa1: 3.48

pKa2: NA

Molecular charge: -1<smiles>O=[N+]([O-])c1ccc([O-])c2ncccc12</smiles>

Compounds: Nitroxoline

CAS Nr.: 4008-48-4

DockingScores: -6.87

Electro: 3

Unpolar: 3

pKa1: 6.89

pKa2: NA

Molecular charge: -1

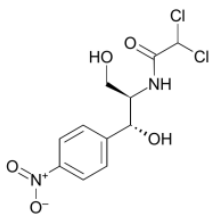

Compounds: Chloramphenicol

CAS Nr.: 56-75-7

DockingScores: -6.84

Electro: 3

Unpolar: 9

pKa1: 8.69

pKa2: 13.55

Molecular charge: 0<smiles>Nc1ccc(C(=O)O)c(O)c1</smiles><smiles>Cc1ccc(C(=O)[O-])cc1</smiles>

Compounds: Sodium 4-aminosalicylate CAS Nr.: 133-10-8

DockingScores: -6.95

Electro: 3

Unpolar: 2

pKa1: 3.68

pKa2: 13.22

Molecular charge: -1

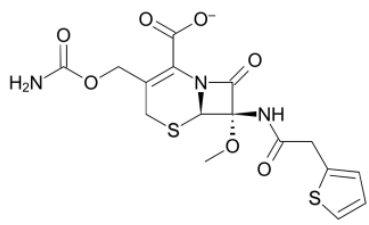

Compounds: Cefoxitin sodium salt

CAS Nr. : 33564-30-6

DockingScores: -6.9

Electro: 3

Unpolar: 11

pKa1: 3.59

pKa2: 10.97

Molecular charge: -1<smiles>COC(=O)COc1ccccc1C</smiles>

Compounds: (2-Methylphenoxy)acetic acid

CAS Nr.: 1878-49-5

DockingScores: -6.86

Electro: 3

Unpolar: 5

pKa1: 3.88

pKa2: NA

Molecular charge: -1

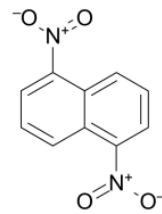

Compounds: 1,5-Dinitronaphthalene

CAS Nr.: 605-71-0

DockingScores: -6.83

Electro: 3

Unpolar: 5

pKa1: NA

pKa2: NA

Molecular charge: 0
Compounds: 4-Methylbenzoic acid CAS Nr: : 99-94-5

DockingScores: -6.95

Electro: 3

Unpolar: 4

pKa1: 4.26

pKa2: NA

Molecular charge: -1<smiles>Nc1ccc(O)c(C(=O)[O-])c1</smiles>

Compounds: 5-Aminosalicylic acid

CAS Nr: : 89-57-6

DockingScores: -6.9

Electro: 3

Unpolar: 2

pKa1: 2.02

pKa2: 15.48

Molecular charge: -1

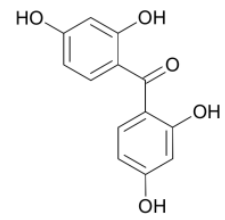

Compounds: $2,2^{\prime}, 4,4^{\prime}-$

Tetrahydroxybenzophenone

CAS Nr.: 131-55-5

DockingScores: -6.84

Electro: 3

Unpolar: 6

pKa1: 6.75

pKa2: 7.38

Molecular charge: 0<smiles>O=S(=O)([O-])c1cc(Cl)c(Cl)cc1Cl</smiles>

Compounds: 2,4,5-

Trichlorobenzenesulfonic acid

CAS Nr: 6378-25-2

DockingScores: -6.8

Electro: 3

Unpolar: 8

pKa1: -3.61

pKa2: NA

Molecular charge: -1

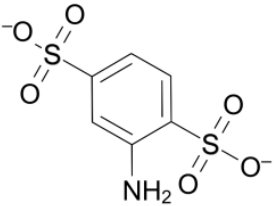

Compounds: 2-Aminobenzene-1,4disulfonic acid

CAS Nr.: 98-44-2

DockingScores: -6.92

Electro: 3

Unpolar: 4

pKa1: -3.14

pKa2: -2.3

Molecular charge: -2<smiles>O=C(O)c1nc(Cl)c(Cl)c(Cl)c1Cl</smiles>

Compounds: 3,4,5,6-

Tetrachloropyridine-2-carboxylic acid

CAS Nr: : 10469-09-7

DockingScores: -6.9

Electro: 3

Unpolar: 7

pKa1: 3.07

pKa2: NA

Molecular charge: -1<smiles>CC1=C(C(=O)[O-])N2C(=O)[C@@H](NC(=O)[C@H]([NH3+])c3ccc(O)cc3)[C@H]2SC1</smiles>

Compounds: Cefadroxil

CAS Nr: : 50370-12-2

DockingScores: -6.84

Electro: 3

Unpolar: 13

pKa1: 3.45

pKa2: 9.48

Molecular charge: 0<smiles>O=C([O-])c1cc(C(=O)[O-])cc(C(=O)[O-])c1</smiles>

Compounds: 1,3,5-Benzenetricarboxylic acid

CAS Nr. : 554-95-0

DockingScores: -6.79

Electro: 3

Unpolar: 2

pKa1: 3.14

pKa2: 3.85

Molecular charge: -3 
<smiles>C[C@H](N[C@@H](CCc1ccccc1)C(=O)[O-])C(=O)N1CCC[C@H]1C(=O)O</smiles><smiles>CC[C@H](Cc1c(I)cc(I)c([O-])c1I)C(=O)O</smiles>

Compounds: Enalapril acid CAS Nr.: 76420-72-9 DockingScores: -6.74 Electro: 3

Unpolar: 8

pKa1: 3.13

pKa2: 3.96

Molecular charge: -1<smiles>NNc1ccc(C(=O)O)cc1</smiles>

Compounds: 4-Hydrazinobenzoic acid hydrochloride

CAS Nr.: 24589-77-3

DockingScores: -6.73

Electro: 3

Unpolar: 3

pKa1: 4.34

pKa2: 19.14

Molecular charge: -1<smiles>Nc1c(Cl)c(Cl)nc(C(=O)[O-])c1Cl</smiles>

Compounds: Picloram

CAS Nr.: 1918-02-1

DockingScores: -6.62

Electro: 3

Unpolar: 4

pKa1: 3.9

pKa2: NA

Molecular charge: -1<smiles>COC(=O)c1cc(C(=O)[O-])cc([N+](=O)[O-])c1</smiles>

Compounds: 5-Nitroisophthalic acid CAS Nr.: 618-88-2

DockingScores: -6.53

Electro: 3

Unpolar: 2

pKa1: 2.94

pKa2: 3.78

Molecular charge: -2
Compounds: Iophenoxic acid

CAS Nr.: 96-84-4

DockingScores: -6.74

Electro: 3

Unpolar: 9

pKa1: 2.43

pKa2: 6.78

Molecular charge: -2

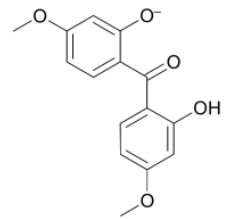

Compounds: 2,2'-Dihydroxy-4,4'-

dimethoxybenzophenone

CAS Nr: : 131-54-4

DockingScores: -6.71

Electro: 3

Unpolar: 4

pKa1: 6.74

pKa2: 7.39

Molecular charge: -1<smiles>O=C(O)c1cc(Cl)cc(Cl)c1</smiles>

Compounds: 3,5-Dichlorobenzoic acid

CAS Nr.: 51-36-5

DockingScores: -6.6

Electro: 3

Unpolar: 5

pKa1: 3.72

pKa2: NA

Molecular charge: -1<smiles>Cc1ccc([N+](=O)[O-])cc1S(=O)(=O)[O-]</smiles>

Compounds: 2-Methyl-5nitrobenzenesulfonic acid

CAS Nr. : 121-03-9

DockingScores: -6.5

Electro: 3

Unpolar: 3

pKa1: -2.72

pKa2: NA

Molecular charge: -1<smiles>Cc1cc(N)c(S(=O)(=O)[O-])cc1Cl</smiles>

Compounds: 2-Amino-5-chloro-4 methylbenzenesulfonic acid

CAS Nr.: 88-53-9

DockingScores: -6.74

Electro: 3

Unpolar: 8

pKa1: -4.01

pKa2: 16.58

Molecular charge: -1<smiles>CCOc1ccccc1C(N)=O</smiles>

Compounds: 2-Ethoxybenzamide

CAS Nr: : 938-73-8

DockingScores: -6.7

Electro: 3

Unpolar: 5

pKa1: 13.68

pKa2: NA

Molecular charge: 0<smiles>Cc1ccc2c(Cl)cc(Cl)c([O-])c2n1</smiles>

Compounds: 5,7-Dichloro-8-hydroxy-2- Compounds: Nilutamide

methylquinoline

CAS Nr: $72-80-0$

DockingScores: -6.58

Electro: 3

Unpolar: 3

pKa1: 6.99

pKa2: NA

Molecular charge: -1<smiles>O=C([O-])c1cc(Cl)cc(Cl)c1O</smiles>

Compounds: 3,5-Dichlorosalicylic acid CAS Nr.: 320-72-9

DockingScores: -6.49

Electro: 3

Unpolar: 3

pKa1: 2.42

pKa2: 9.86

Molecular charge: -1

Molecular charge: -1<smiles>Cc1cc(S(=O)(=O)[O-])c(N)cc1Cl</smiles>

Compounds: 2-Amino-4-chloro-5methylbenzenesulfonic acid CAS Nr.: 88-51-7

DockingScores: -6.74

Electro: 3

Unpolar: 8

pKa1: -4.04

pKa2: 16.78

Molecular charge: -1<smiles>O=C([O-])c1snc(Cl)c1Cl</smiles>

Compounds: 3,4-Dichloro-5isothiazolecarboxylic acid

CAS Nr.: 18480-53-0

DockingScores: -6.62

Electro: 3

Unpolar: 4

pKa1: 2.9

pKa2: NA

Molecular charge: -1<smiles>CC1(C)NC(=O)N(c2ccc([N+](=O)[O-])c(C(F)(F)F)c2)C1=O</smiles>

DockingScores: -6.58

Electro: 3

Unpolar: 8

pKa1: 15.01

pKa2: NA

Molecular charge: 0

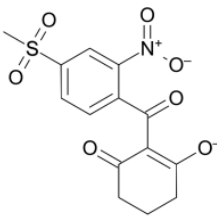

Compounds: Mesotrione

CAS Nr.: 104206-82-8

DockingScores: -6.32

Electro: 3

Unpolar: 5

pKa1: 3.7

pKa2: 19.64 
Table S7. Information of the previously confirmed TTR binders in the potential TTR binders (refined hits) proposed by the virtual screening protocol

\begin{tabular}{|c|c|c|}
\hline Compounds & CAS Nr. & Chemical Class \\
\hline Diflunisal $^{14}$ & $22494-42-4$ & Pharmaceutical \\
\hline Niflumic acid $^{14}$ & $4394-00-7$ & Pharmaceutical \\
\hline Aceclofenac $^{14}$ & $89796-99-6$ & Pharmaceutical \\
\hline Meclofenamate sodium ${ }^{14}$ & $6385-02-0$ & Pharmaceutical \\
\hline Tolcapone $^{42}$ & $134308-13-7$ & Pharmaceutical \\
\hline 2,4,5-Trichlorophenoxyacetic acid ${ }^{5}$ & $93-76-5$ & Herbicide \\
\hline $2,2^{\prime}, 4,4^{\prime}$-Tetrahydroxybenzophenone ${ }^{5}$ & $131-55-5$ & UV filter \\
\hline Quercetin dihydrate $^{43}$ & $6151-25-3$ & Food supplement \\
\hline 3,3',5,5'-Tetraiodothyroacetic acid ${ }^{44}$ & $67-30-1$ & Thyroxine derivative \\
\hline $3,3^{\prime}, 5,5^{\prime}$-Tetrachlorobisphenol $\mathrm{A}^{6}$ & $79-95-8$ & Flame retardant \\
\hline $3,3^{\prime}, 5,5^{\prime}$-Tetrabromobisphenol A ${ }^{6}$ & $79-94-7$ & Flame retardant \\
\hline
\end{tabular}


Table S8. Information of the industrial compounds tested by the isothermal titration calorimetry (ITC) and human plasma assay.

\begin{tabular}{|c|c|c|c|c|c|}
\hline Compounds (Abbreviations) & CAS Nr. & $\mathrm{pKa}^{\mathrm{a}}$ & $\log \mathrm{P}^{\mathrm{b}}$ & Purity & Provider \\
\hline $3,3^{\prime}, 5,5^{\prime}-$ Tetraiodothyronine (T4) & $51-48-9$ & 2.12 & 4.72 & $98 \%$ & $\begin{array}{l}\text { Sigma-Aldrich Sweden AB } \\
\text { (Stockholm, Sweden) }\end{array}$ \\
\hline $\begin{array}{l}\text { 2-Nitro-5-(2-chloro-4- } \\
\text { trifluoromethylphenoxy)benzoic acid } \\
\text { (Acifluorfen) }\end{array}$ & $50594-66-6$ & 1.93 & 3.77 & $98 \%$ & $\begin{array}{l}\text { Sigma-Aldrich Sweden AB } \\
\text { (Stockholm, Sweden) }\end{array}$ \\
\hline $\begin{array}{l}\text { 2,2'-dihydroxy-4,4'- } \\
\text { dimethoxybenzophenone (BP6) }\end{array}$ & $131-54-4$ & 6.81 & 4.77 & $98 \%$ & $\begin{array}{l}\text { Sigma-Aldrich Sweden AB } \\
\text { (Stockholm, Sweden) }\end{array}$ \\
\hline $\begin{array}{l}\text { 4,4'-Dihydroxydiphenyl sulfone } \\
\text { (BPS) }\end{array}$ & $80-09-1$ & 7.64 & 2.14 & $98 \%$ & $\begin{array}{l}\text { Sigma-Aldrich Sweden AB } \\
\text { (Stockholm, Sweden) }\end{array}$ \\
\hline $\begin{array}{l}\text { 2-(3-chloro-2-methylanilino)pyridine- } \\
\text { 3-carboxylic acid (Clonixin) }\end{array}$ & $17737-65-4$ & 1.69 & 4.71 & $98 \%$ & $\begin{array}{l}\text { Sigma-Aldrich Sweden AB } \\
\text { (Stockholm, Sweden) }\end{array}$ \\
\hline 2,6-Dinitro-p-cresol (DNPC) & $609-93-8$ & 2.98 & 2.07 & $98 \%$ & $\begin{array}{l}\text { TCI Germany GmbH } \\
\text { (Eschborn, Germany) }\end{array}$ \\
\hline Diphenolic acid (DPA) & $126-00-1$ & 4.66 & 2.83 & $95 \%$ & $\begin{array}{l}\text { Sigma-Aldrich Sweden AB } \\
\text { (Stockholm, Sweden) }\end{array}$ \\
\hline $\begin{array}{l}\text { 2-[(4-Amino-3,5-dichloro-6-fluoro-2- } \\
\text { pyridinyl)oxy]acetic acid } \\
\text { (Fluroxypyr) }\end{array}$ & $69377-81-7$ & 2.22 & 1.85 & $98 \%$ & $\begin{array}{l}\text { Sigma-Aldrich Sweden AB } \\
\text { (Stockholm, Sweden) }\end{array}$ \\
\hline L- $\gamma$-Glutamyl-p-nitroanilide (GPNA) & $67953-08-6$ & 2.21 & 0.54 & $98 \%$ & $\begin{array}{l}\text { Sigma-Aldrich Sweden AB } \\
\text { (Stockholm, Sweden) }\end{array}$ \\
\hline $\begin{array}{l}\text { 2-[4-(Methylsulfonyl)-2- } \\
\text { nitrobenzoyl]-1,3-cyclohexanedione } \\
\text { (Mesotrione) }\end{array}$ & $104206-82-8$ & 2.98 & -0.98 & $97 \%$ & $\begin{array}{lr}\text { LGC Standards } & \text { GmbH } \\
\text { (Wesel, Germany) } & \end{array}$ \\
\hline $\begin{array}{l}\text { 3,5,6-Trichloro-4-aminopicolinic acid } \\
\text { (Picloram) }\end{array}$ & $1918-02-1$ & 1.93 & 2.00 & $98 \%$ & $\begin{array}{l}\text { Sigma-Aldrich Sweden AB } \\
\text { (Stockholm, Sweden) }\end{array}$ \\
\hline $\begin{array}{l}\text { 1-(4-Sulfophenyl)-3-carboxy-5- } \\
\text { pyrazolone (PyT) }\end{array}$ & $118-47-8$ & -2.65 & -2.61 & $98 \%$ & $\begin{array}{l}\text { Sigma-Aldrich Sweden AB } \\
\text { (Stockholm, Sweden) }\end{array}$ \\
\hline $\begin{array}{l}\text { 3,5,6-Trichloro-2-pyridinyloxyacetic } \\
\text { acid (Triclopyr) }\end{array}$ & $55335-06-3$ & 2.26 & 2.35 & $98 \%$ & $\begin{array}{l}\text { Sigma-Aldrich Sweden AB } \\
\text { (Stockholm, Sweden) }\end{array}$ \\
\hline $2,2^{\prime}, 4,4^{\prime}$-Tetrahydroxybenzophenone & $131-55-5$ & 6.98 & 3.09 & $97 \%$ & $\begin{array}{l}\text { Sigma-Aldrich Sweden AB } \\
\text { (Stockholm, Sweden) }\end{array}$ \\
\hline Perfluorooctanoic acid (PFOA) & $335-67-1$ & 0.50 & 6.44 & $96 \%$ & $\begin{array}{l}\text { Sigma-Aldrich Sweden AB } \\
\text { (Stockholm, Sweden) }\end{array}$ \\
\hline Perfluorooctanesulfonic acid (PFOS) & $1763-23-1$ & -3.27 & 4.51 & $98 \%$ & $\begin{array}{l}\text { Sigma-Aldrich Sweden AB } \\
\text { (Stockholm, Sweden) }\end{array}$ \\
\hline
\end{tabular}

${ }^{\mathrm{a}} \mathrm{pKa}$ values and ${ }^{\mathrm{b}} \operatorname{LogP}\left(\right.$ at $\left.25^{\circ} \mathrm{C}\right)$ of the compounds were collected at the SciFinder database. ${ }^{45}$ 
Table S9. Thermodynamic parameters of the active compounds and T4 binding to TTR.

\begin{tabular}{|c|c|c|c|c|c|c|}
\hline Compounds & CAS Nr. & $\mathrm{K}_{\mathrm{d}}(\mu \mathrm{M})$ & $\mathrm{N}$ & $\begin{array}{l}\Delta \mathrm{H} \\
(\mathrm{kcal} / \mathrm{mol})\end{array}$ & $\begin{array}{l}-\mathrm{T} \Delta \mathrm{S} \\
(\mathrm{kcal} / \mathrm{mol})\end{array}$ & $\begin{array}{l}\Delta \mathrm{G} \\
(\mathrm{kcal} / \mathrm{mol})\end{array}$ \\
\hline $\mathrm{T} 4^{\mathrm{a}}$ & $51-48-9$ & 0.09 & $0.7 \pm 0.04$ & $-5.6 \pm 0.66$ & -1.57 & -7.2 \\
\hline BPS & $80-09-1$ & 52 & $1.2 \pm 0.03$ & $-5.0 \pm 0.17$ & -0.80 & -5.8 \\
\hline Clonixin & $17737-65-4$ & 0.26 & $0.9 \pm 0.01$ & $-10.2 \pm 0.64$ & 1.2 & -9.0 \\
\hline Dinitro-p-cresol & $609-93-8$ & 1.3 & $0.9 \pm 0.01$ & $-3.3 \pm 0.041$ & -4.8 & -8.1 \\
\hline Fluroxypyr & $69377-81-7$ & 45 & $1.0 \pm 0.02$ & $-5.0 \pm 0.13$ & -0.9 & -5.9 \\
\hline Mesotrione & $\begin{array}{l}104206-82- \\
8\end{array}$ & 99 & $1.2 \pm 0.05$ & $-6.5 \pm 0.49$ & 1.1 & -5.4 \\
\hline Picloram & 1918-02-1 & 63 & $1.1 \pm 0.04$ & $-2.3 \pm 0.15$ & -3.5 & -5.8 \\
\hline Triclopyr & $55335-06-3$ & 4.6 & $0.8 \pm 0.01$ & $-6.9 \pm 0.05$ & -0.36 & -7.2 \\
\hline
\end{tabular}

The full name of the parameters in the table are disassociation constant $(\mathrm{Kd})$, enthalpy changes $(\Delta \mathrm{H})$, binding stoichiometry $(\mathrm{N})$, Gibbs energy changes $(\Delta \mathrm{G})$, and entropy changes $(\Delta \mathrm{S})$. ${ }^{\mathrm{a}}$ The binding affinity of T4 was an approximate value due to its thermo-instability and poor solubility in the ITC buffer. The approximated binding affinity is similar to the value of $84 \mathrm{nM}$ reported previously. ${ }^{46}$ 

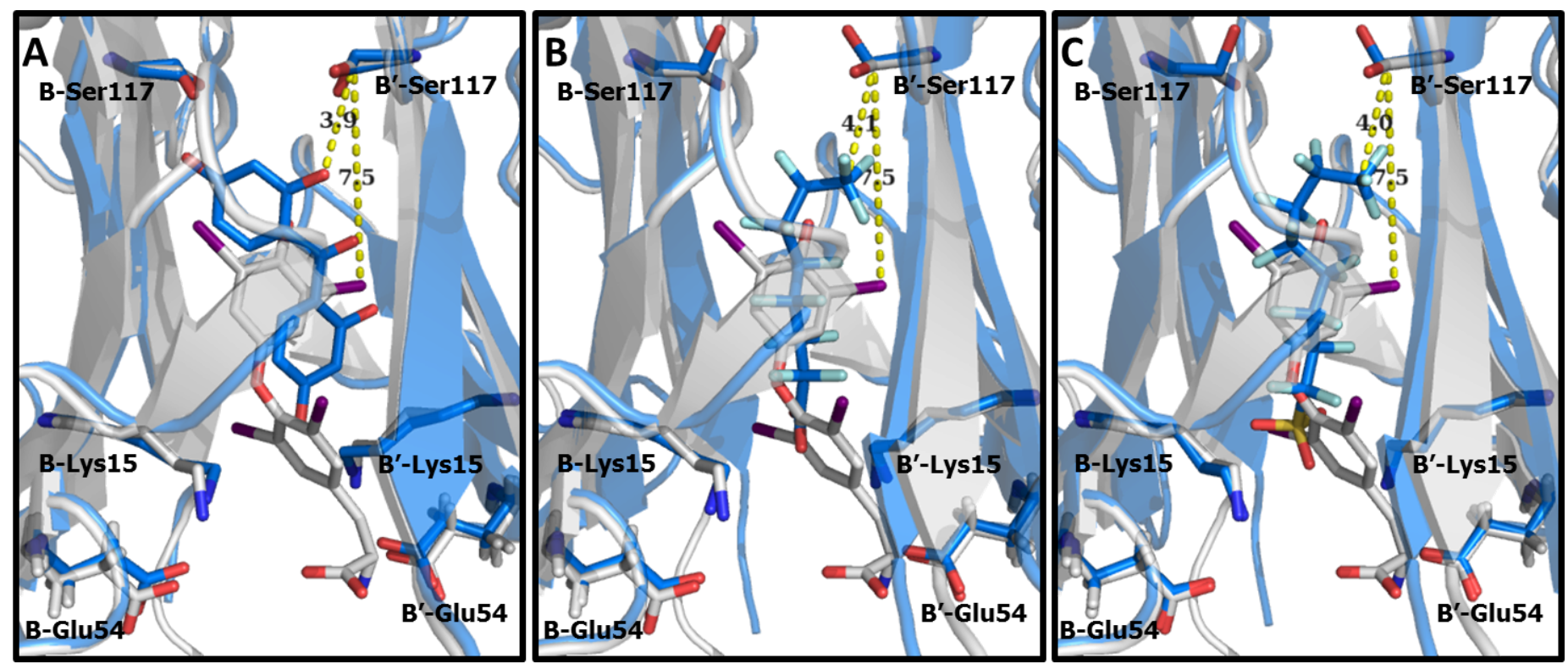

Figure S1. Comparisons of binding conformations between (A) BP2 (Blue), (B) PFOA (Blue), (C) PFOS (Blue), and T4 (PDB ID: 2ROX, Grey). The three ligands bind deeper into the TBS, due to their small molecular volume, i.e. BP2 $\left(178 \AA^{3}\right)$, PFOA $\left(224 \AA^{3}\right)$, and PFOS $\left(249 \AA^{3}\right)$, comparing with T4 $\left(315 \AA^{3}\right)$. 

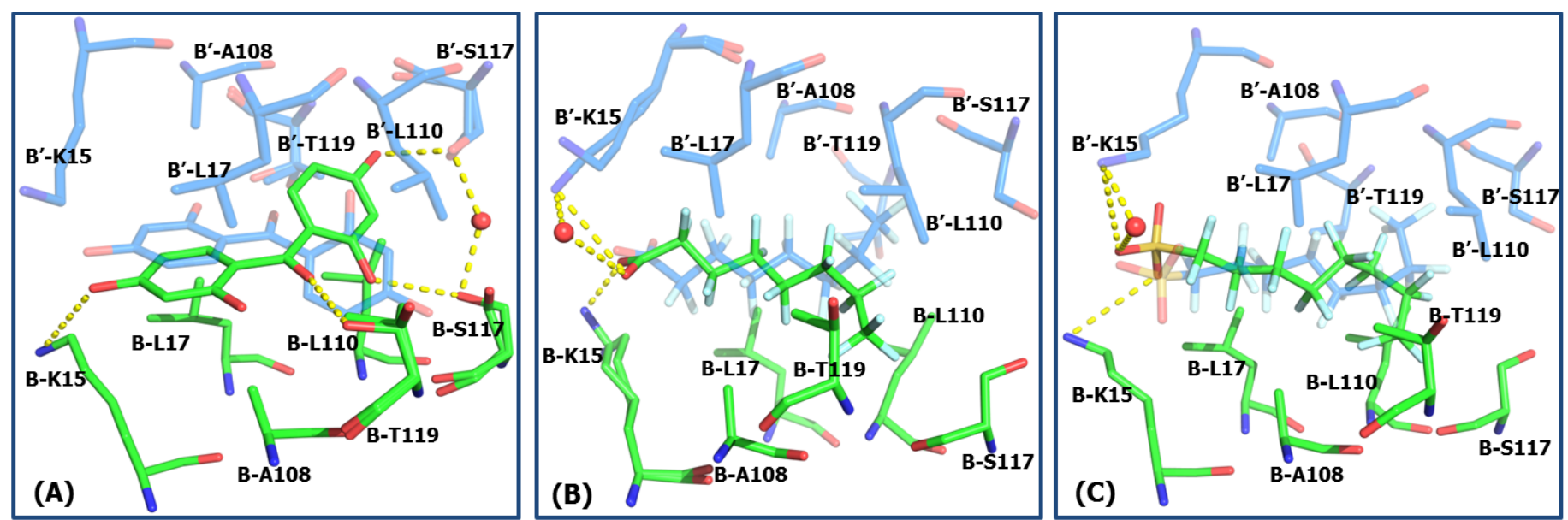

Figure S2. Close-up views of the molecular interactions between TTR and (A) BP2, (B) PFOA, and (C) PFOS in the TBS situated at the BB' homomoner interface. The possible electrostatic interactions were shown in yellow bash lines. 

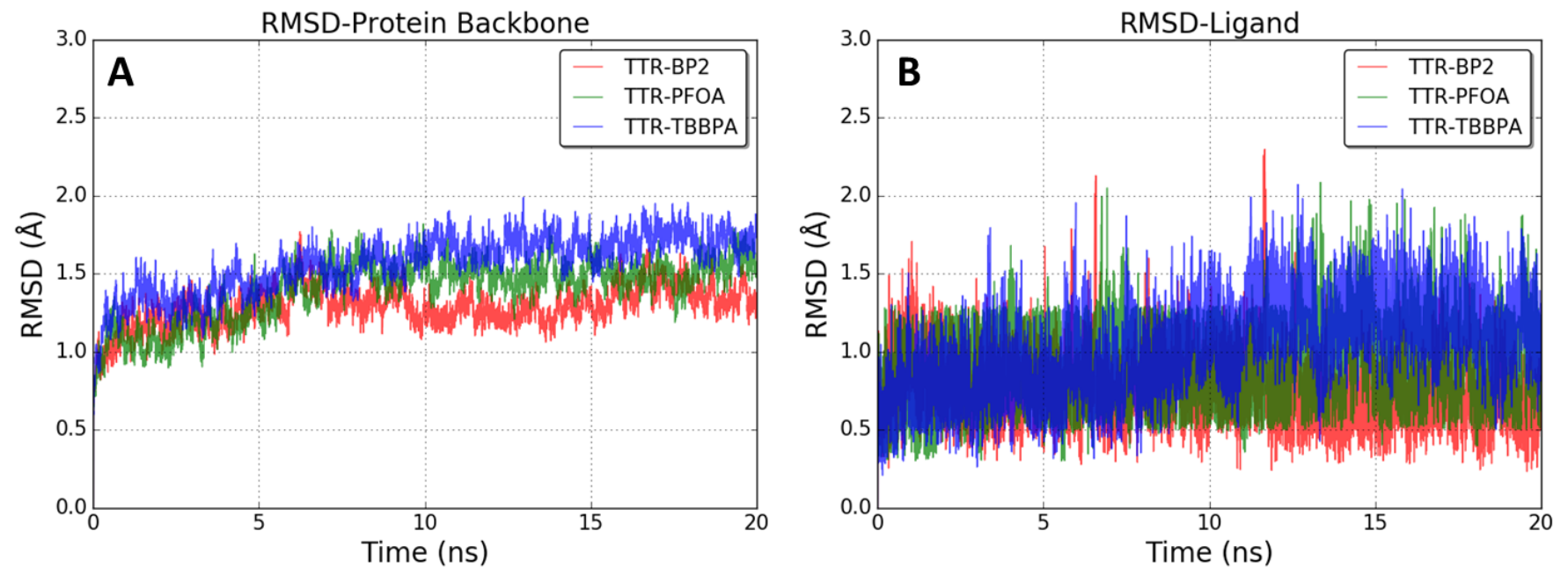

Figure S3. RMSDs of (A) protein backbone and (B) the coligands (the one used for MM-GBSA calculations) during molecular dynamics simulations of the TTR complexes structures with BP2, PFOA, and TBBPA. 

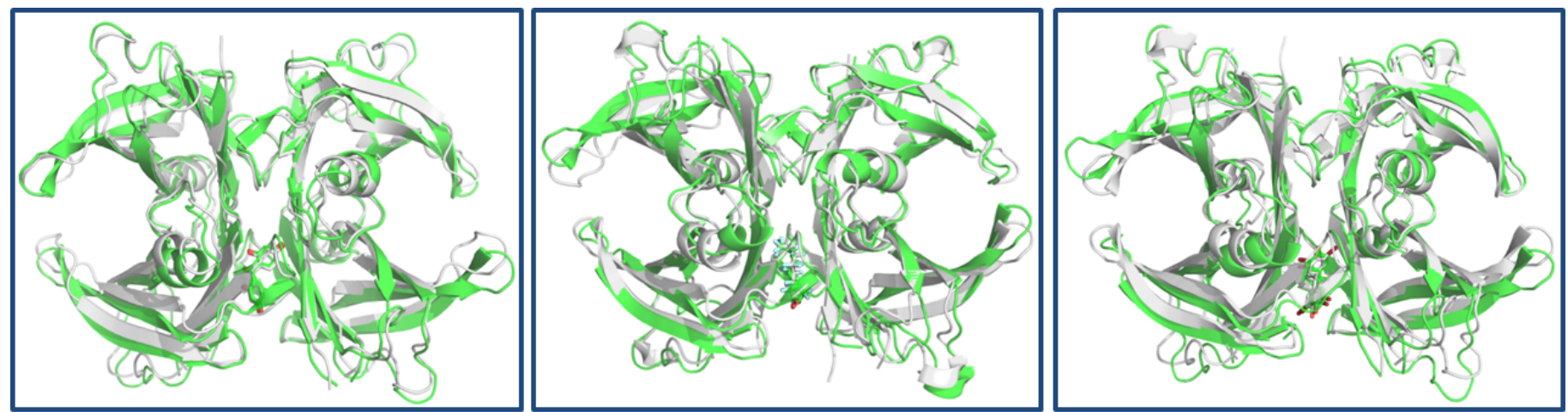

(a)

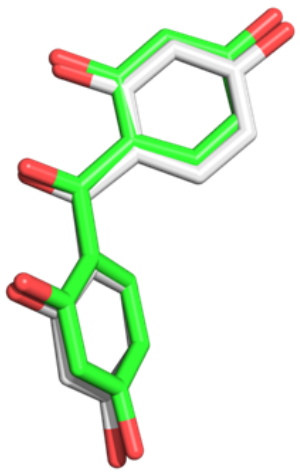

(b)

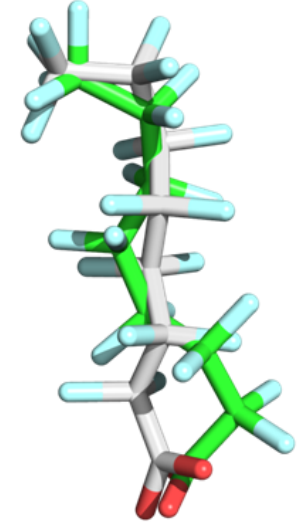

(c)

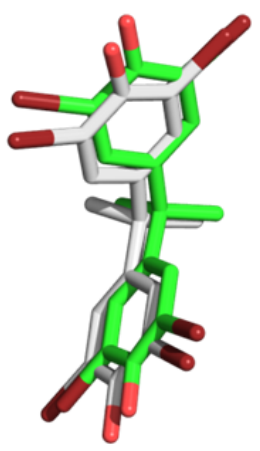

Figure S4. Comparison of the backbones and ligand poses (the one used for MM-GBSA calculations) between the crystal structures and MD-averaged structures (the last 2 ns) of: (a) BP2, (b) PFOA, and (c) TBBPA. The crystal structures and MD average structures are shown in grey and green, respectively. 


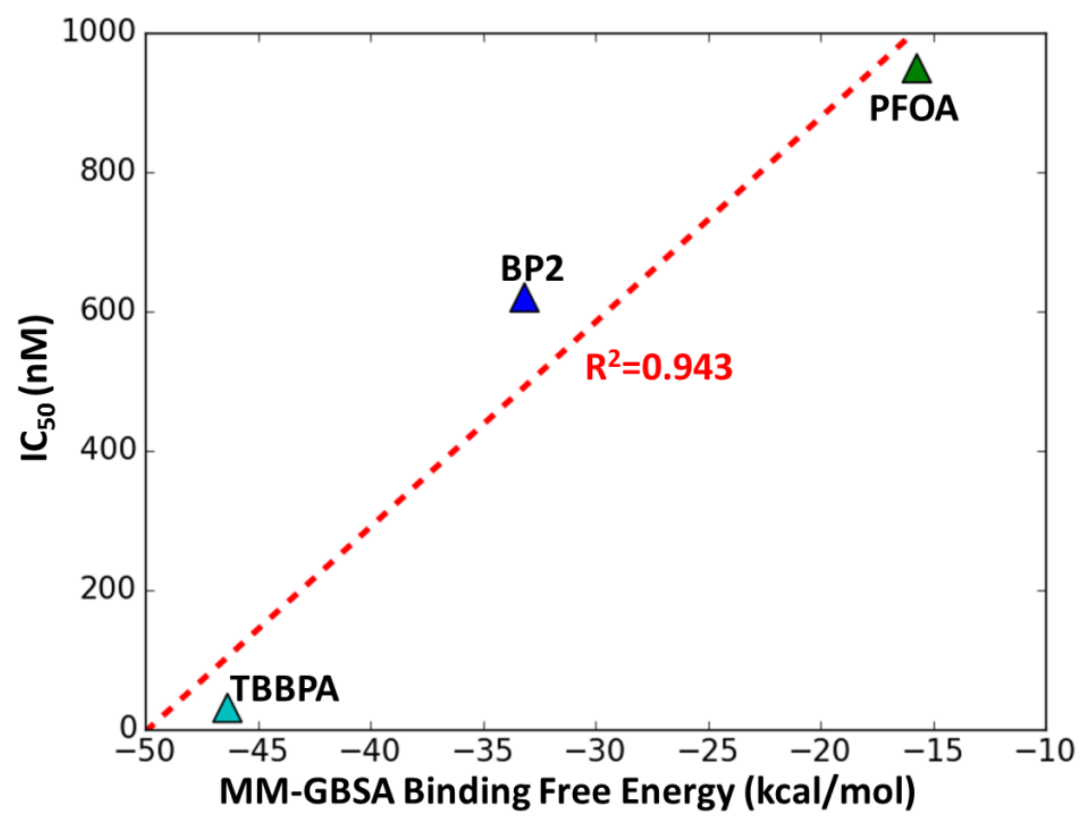

Figure S5. Correlations between the MM-GBSA calculated binding free energies and the previously in vitro measured potencies of BP2, PFOA, and TBBPA. ${ }^{5,47,48}$. 


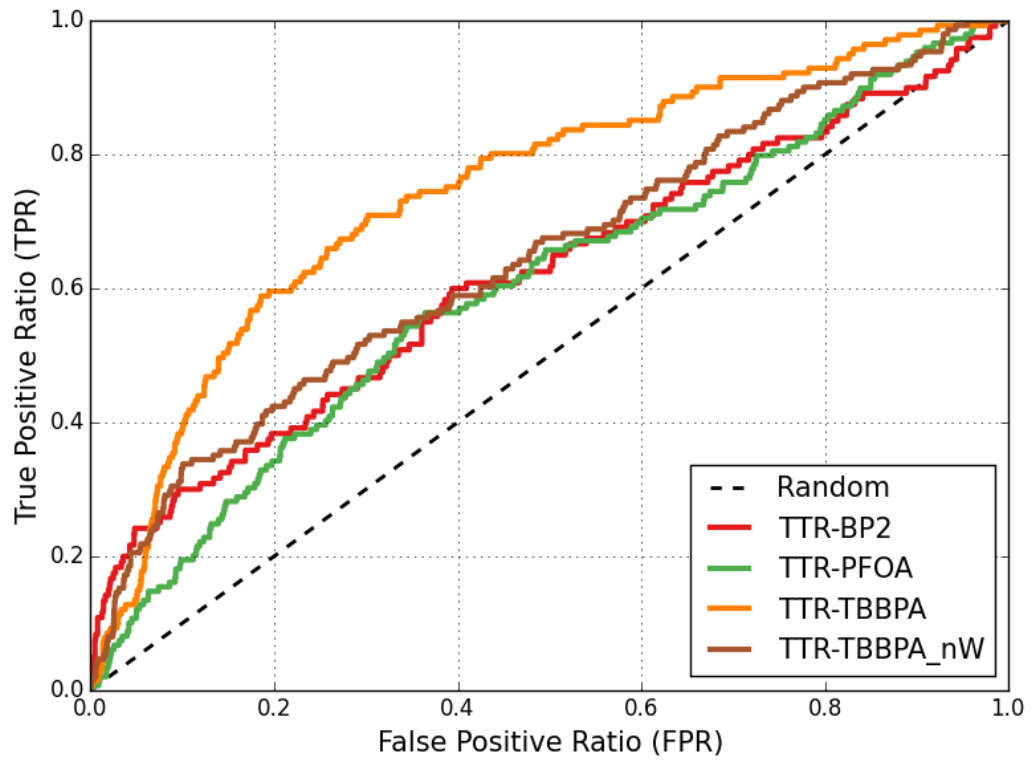

Figure S6. The receiver operating characteristic (ROC) curves of molecular docking models using four TTR complex structures. The TTR-TBBPA_nW structure refers to the TTR-TBBPA complex structure with the water molecules in the TBS removed. 

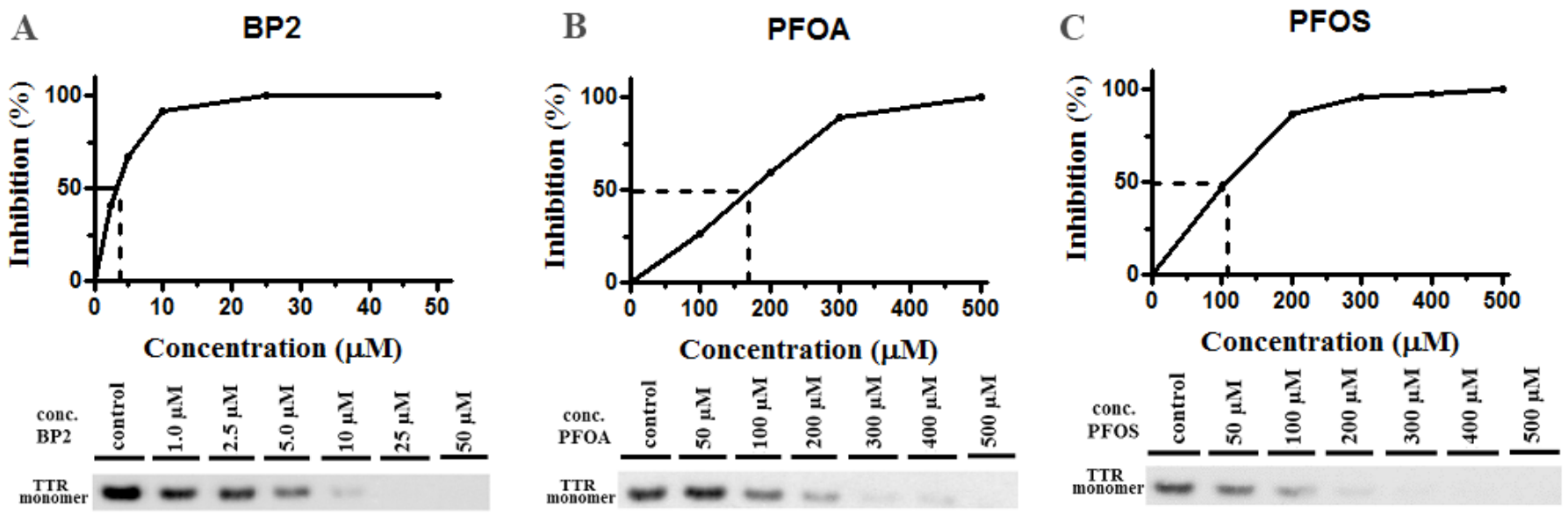

Figure S7. Determining the selectivity of (A) BP2, (B) PFOA, (C) PFOS for TTR in human plasma. BP2, PFOA, and PFOS were added at different concentrations to human plasma from a single healthy donor and incubated for $2 \mathrm{~h}$. The conversion of TTR into monomer was initiated by the addition of urea at a final concentration of 4.0 M. The monomeric TTR was monitored by western blot and quantified with the ImageJ 2.0 software. The inhibitory concentration at $50 \%$ in plasma (IC50) was estimated for (A) BP2; (B) PFOA;

(C) PFOS. The results have revealed that BP2 is an efficient binder to TTR in plasma and displays an IC50 corresponding to $4 \pm 0.5 \mu \mathrm{M}$, comparing to PFOA and PFOS, which are required at $175 \pm 5 \mu \mathrm{M}$ and $103 \pm 7$ $\mu \mathrm{M}$ concentrations in plasma to reach IC50. 


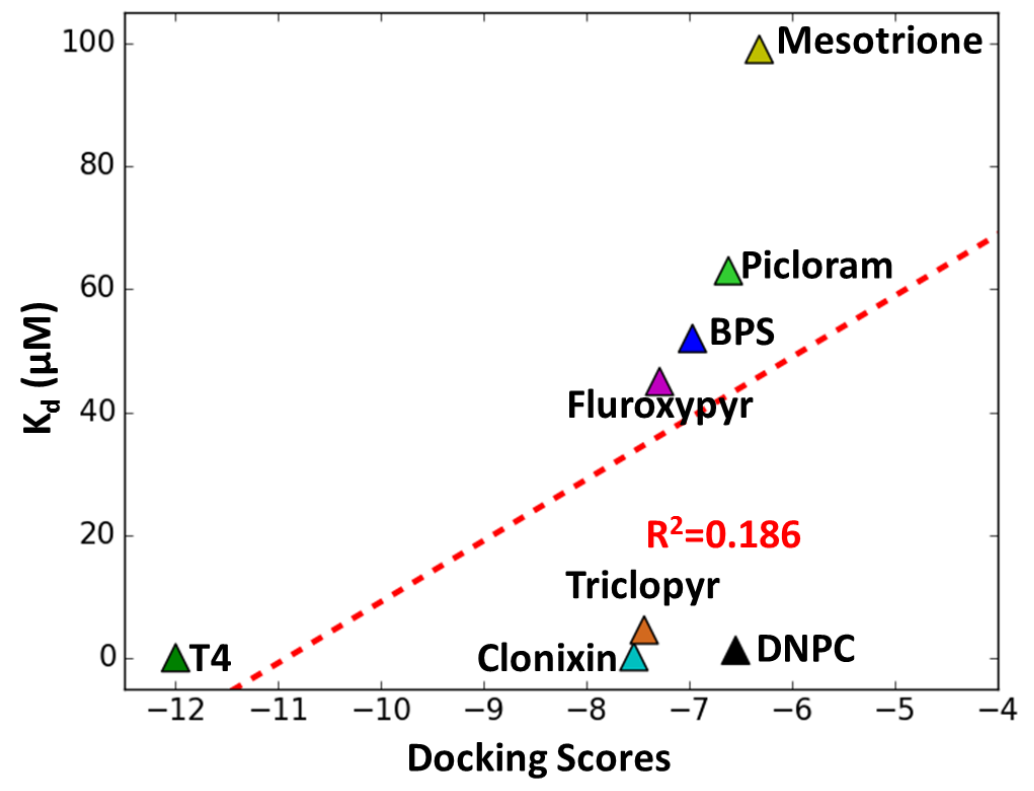

Figure S8. Correlations between the docking scores and the ITC measured binding affinities $\left(\mathrm{K}_{\mathrm{d}}\right)$ of the active compounds. 
<smiles>O=C(O)c1cc(Oc2ccc(C(F)(F)F)cc2Cl)ccc1[N+](=O)[O-]</smiles>

2-Nitro-5-(2-chloro-4-trifluoromethylphenoxy)benzoic acid Abbrev. : Acifluorfen CAS Nr. : 50594-66-6<smiles>CC(CCC(=O)O)(c1ccc(O)cc1)c1ccc(O)cc1</smiles>

Diphenolic acid Abbrev. : DPA CAS Nr. : 126-00-1<smiles>N[C@@H](CCC(=O)Nc1ccc([N+](=O)[O-])cc1)C(=O)O</smiles>

L-y-Glutamyl-p-nitroanilide Abbrev. : GPNA CAS Nr. : 67953-08-6<smiles>COc1ccc(C(=O)c2ccc(OC)cc2O)c(O)c1</smiles>

2,2'-dihydroxy-4,4'-dimethoxybenzophenone Abbrev. : BP6

CAS Nr. : 131-54-4

Figure S9. Structures and chemical information of the five false positives identified by ITC. 

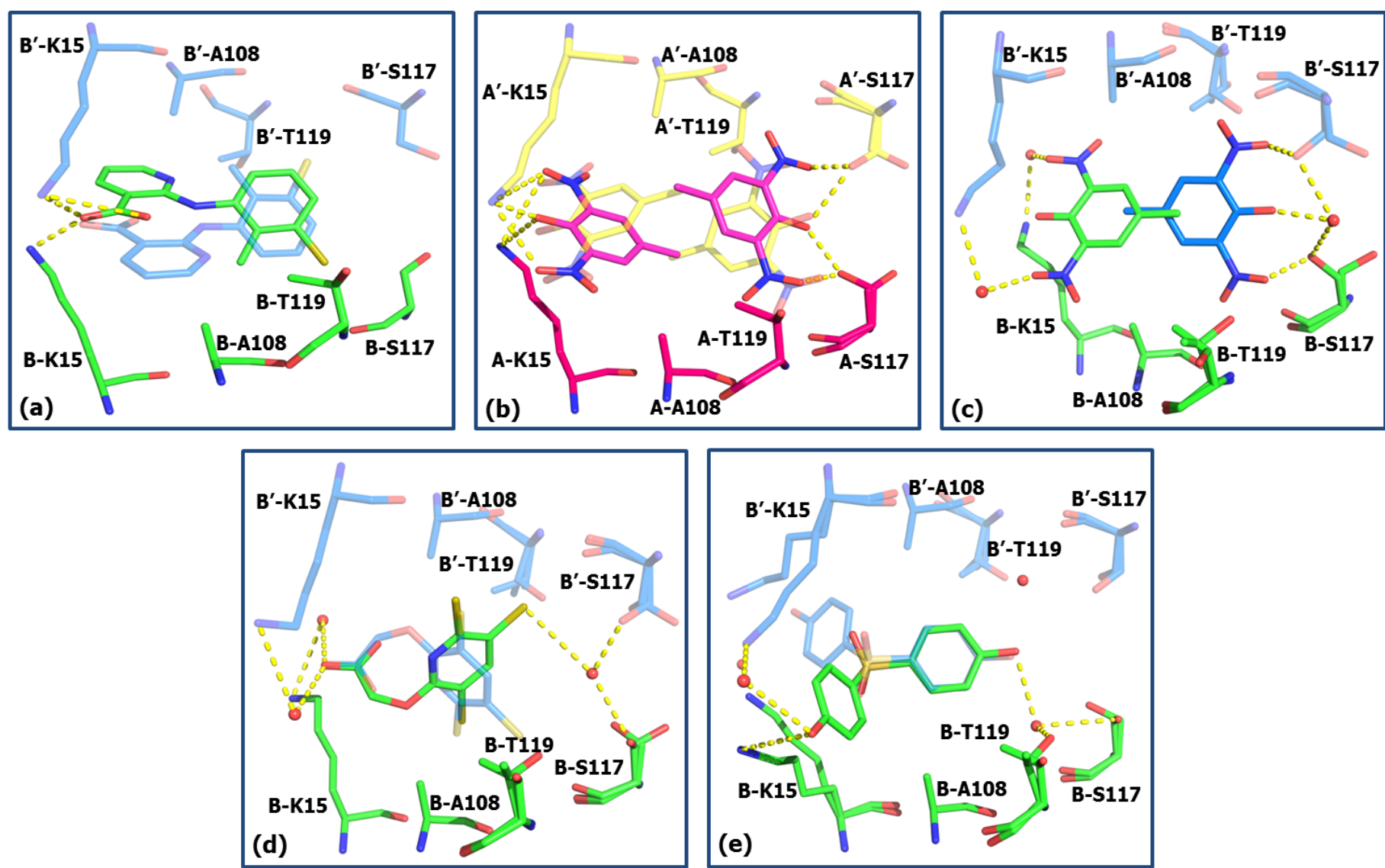

Figure S10. Close-up views of the molecular interactions between TTR and (a) clonixin, (b) DNPC (AA' interface), (c) DNPC, (d) triclopyr, and (e) BPS in the TBS. The possible electrostatic interactions were shown in yellow bash lines. 


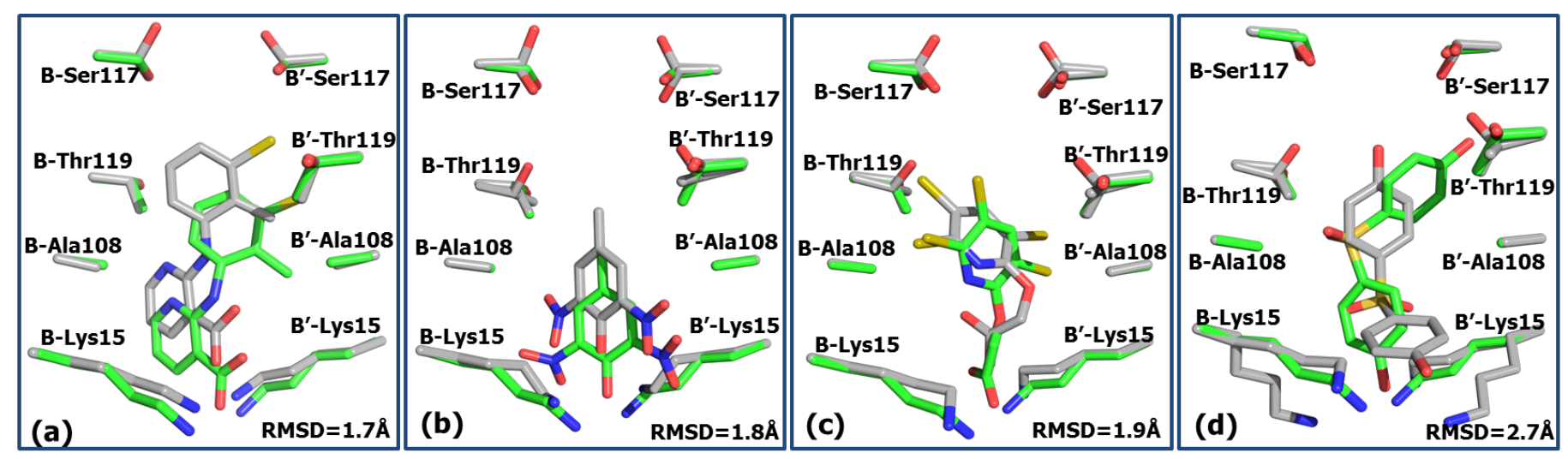

Figure S11. Comparison between crystal conformations (sticks in grey) and docking poses (sticks in green) for (a) clonixin, (b) DNPC, (c) triclopyr, and (d) BPS in the TBS. The RMSDs between crystal conformations and docking poses for the coligands (a) clonixin, (b) DNPC, (c) triclopyr, and (d) BPS are 1.7 $\AA, 1.8 \AA, 1.9 \AA$ and $2.7 \AA$, respectively. 


\section{References}

(1) Browne, P.; Judson, R. S.; Casey, W. M.; Kleinstreuer, N. C.; Thomas, R. S., Screening Chemicals for Estrogen Receptor Bioactivity Using a Computational Model. Environ. Sci. Technol. 2015, 49, (14), 880414.

(2) Rabinowitz, J. R.; Little, S. B.; Laws, S. C.; Goldsmith, M. R., Molecular modeling for screening environmental chemicals for estrogenicity: use of the toxicant-target approach. Chem. Res. Toxicol. 2009, 22, (9), 1594-602.

(3) Freitas, J.; Miller, N.; Mengeling, B. J.; Xia, M.; Huang, R.; Houck, K.; Rietjens, I. M.; Furlow, J. D.; Murk, A. J., Identification of thyroid hormone receptor active compounds using a quantitative highthroughput screening platform. Current chemical genomics and translational medicine 2014, 8, 36-46.

(4) Huang, R.; Xia, M.; Nguyen, D.-T.; Zhao, T.; Sakamuru, S.; Zhao, J.; Shahane, S. A.; Rossoshek, A.; Simeonov, A., Tox21Challenge to build predictive models of nuclear receptor and stress response pathways as mediated by exposure to environmental chemicals and drugs. Frontiers in Environmental Science $2016,3$. (5) Zhang, J.; Kamstra, J. H.; Ghorbanzadeh, M.; Weiss, J. M.; Hamers, T.; Andersson, P. L., In Silico Approach To Identify Potential Thyroid Hormone Disruptors among Currently Known Dust Contaminants and Their Metabolites. Environ. Sci. Technol. 2015, 49, (16), 10099-107.

(6) Weiss, J. M.; Andersson, P. L.; Zhang, J.; Simon, E.; Leonards, P. E.; Hamers, T.; Lamoree, M. H., Tracing thyroid hormone-disrupting compounds: database compilation and structure-activity evaluation for an effect-directed analysis of sediment. Anal. Bioanal. Chem. 2015, 407, (19), 5625-34.

(7) Iakovleva, I.; Begum, A.; Brannstrom, K.; Wijsekera, A.; Nilsson, L.; Zhang, J.; Andersson, P. L.; Sauer-Eriksson, A. E.; Olofsson, A., Tetrabromobisphenol A Is an Efficient Stabilizer of the Transthyretin Tetramer. PLoS One 2016, 11, (4), e0153529.

(8) Schrödinger Protein Preparation Wizard 2015-2, Schrodinger, LLC, New York, NY., 2015.

(9) Frisch, M. J.; Trucks, G. W.; Schlegel, H. B.; Scuseria, G. E., Gaussian, Inc., Wallingford CT. Gaussian 09, Revision E.01 2009.

(10) Case, D. A.; Darden, T. A.; Gusarov, S.; Kovalenko, A.; Kollman, P. A., Amber 2015 (AmberTools15 and Amber14), University of California, San Francisco. 2015.

(11) Petersen, H. G., Accuracy and Efficiency of the Particle Mesh Ewald Method. J. Chem. Phys. 1995, $103,(9), 3668-3679$.

(12) Onufriev, A.; Bashford, D.; Case, D. A., Exploring protein native states and large-scale conformational changes with a modified generalized born model. Proteins 2004, 55, (2), 383-94.

(13) Weiser, J.; Shenkin, P. S.; Still, W. C., Approximate atomic surfaces from linear combinations of pairwise overlaps (LCPO). J. Comput. Chem. 1999, 20, (2), 217-230.

(14) Iakovleva, I.; Brannstrom, K.; Nilsson, L.; Gharibyan, A. L.; Begum, A.; Anan, I.; Walfridsson, M.; Sauer-Eriksson, A. E.; Olofsson, A., Enthalpic Forces Correlate with the Selectivity of TransthyretinStabilizing Ligands in Human Plasma. J. Med. Chem. 2015, 58, (16), 6507-15.

(15) US-DA Fluroxypyr Human Health and Ecological Risk Assessment Final Report; United States Department of Agriculture (USDA) Forest Service, 2009.

(16) Wang, L.; Xu, J.; Zhao, P.; Pan, C., Dissipation and residues of fluroxypyr-meptyl in rice and environment. Bull. Environ. Contam. Toxicol. 2011, 86, (4), 449-53.

(17) Ulen, B. M.; Larsbo, M.; Kreuger, J. K.; Svanback, A., Spatial variation in herbicide leaching from a marine clay soil via subsurface drains. Pest Manag Sci 2014, 70, (3), 405-14.

(18) Cederlund, H.; Borjesson, E.; Jonsson, E.; Thierfelder, T., Degradation and leaching of fluroxypyr after application to railway tracks. J. Environ. Qual. 2012, 41, (6), 1884-92.

(19) Lazartigues, A.; Fratta, C.; Baudot, R.; Wiest, L.; Feidt, C.; Thomas, M.; Cren-Olive, C., Multiresidue method for the determination of 13 pesticides in three environmental matrices: water, sediments and fish muscle. Talanta 2011, 85, (3), 1500-7.

(20) Rolston, M. P.; Devantier, B. P., Alternative Herbicides to 2,4,5-T for Gorse Control. New Zealand Journal of Experimental Agriculture 1983, 11, (1), 91-94.

(21) US-EPA R.E.D. FACTS Picloram; Prevention, Pesticides and Toxic Substances (7508W); United States Environmental Protection Agency (EPA), 1995.

(22) Fairchild, J. F.; Feltz, K. P.; Sappington, L. C.; Allert, A. L.; Nelson, K. J.; Valle, J., An ecological risk assessment of the acute and chronic toxicity of the herbicide picloram to the threatened bull trout (Salvelinus 
confluentus) and the rainbow trout (Onchorhyncus mykiss). Arch. Environ. Contam. Toxicol. 2009, 56, (4), 761-9.

(23) Reuber, M. D., Carcinogenicity of picloram. J. Toxicol. Environ. Health 1981, 7, (2), 207-22.

(24) Tang, L.; Zeng, G. M.; Shen, G. L.; Li, Y. P.; Zhang, Y.; Huang, D. L., Rapid detection of picloram in agricultural field samples using a disposable immunomembrane-based electrochemical sensor. Environ. Sci. Technol. 2008, 42, (4), 1207-12.

(25) Ki, S. J.; Ray, C.; Hantush, M. M., Applying a statewide geospatial leaching tool for assessing soil vulnerability ratings for agrochemicals across the contiguous United States. Water Res. 2015, 77, 107-18.

(26) Zhang, P.; Bui, A.; Rose, G.; Allinson, G., Mixed-mode solid-phase extraction coupled with liquid chromatography tandem mass spectrometry to determine phenoxy acid, sulfonylurea, triazine and other selected herbicides at nanogram per litre levels in environmental waters. J. Chromatogr. A 2014, 1325, 5664.

(27) Mitchell, G.; Bartlett, D. W.; Fraser, T. E.; Hawkes, T. R.; Holt, D. C.; Townson, J. K.; Wichert, R. A., Mesotrione: a new selective herbicide for use in maize. Pest Manag Sci 2001, 57, (2), 120-8.

(28) Piancini, L. D. S.; Guiloski, I. C.; Silva de Assis, H. C.; Cestari, M. M., Mesotrione herbicide promotes biochemical changes and DNA damage in two fish species. Toxicology Reports 2015, 2, 1157-1163.

(29) Lewis, R. W.; Botham, J. W., A review of the mode of toxicity and relevance to humans of the triketone herbicide 2-(4-methylsulfonyl-2-nitrobenzoyl)-1,3-cyclohexanedione. Crit. Rev. Toxicol. 2013, 43, (3), 185-99.

(30) Chen, X.; Li, W.; Wu, Q.; Chen, W.; Han, L., Dissipation and residues of the herbicide mesotrione in maize and soil in open field. Bull. Environ. Contam. Toxicol. 2012, 88, (5), 772-5.

(31) Barchanska, H.; Kowalska, A.; Poloczek, B., Extraction procedures for the study of phytotoxicity and degradation processes of selected triketones in a water ecosystem. Environ. Sci. Pollut. Res. Int. 2014, 21, (6), 4751-8.

(32) Verro, R.; Finizio, A.; Otto, S.; Vighi, M., Predicting pesticide environmental risk in intensive agricultural areas. I: Screening level risk assessment of individual chemicals in surface waters. Environ. Sci. Technol. 2009, 43, (2), 522-9.

(33) Kabsch, W., Xds. Acta Crystallogr. D Biol. Crystallogr. 2010, 66, (Pt 2), 125-32.

(34) Winn, M. D.; Ballard, C. C.; Cowtan, K. D.; Dodson, E. J.; Emsley, P.; Evans, P. R.; Keegan, R. M.; Krissinel, E. B.; Leslie, A. G.; McCoy, A.; McNicholas, S. J.; Murshudov, G. N.; Pannu, N. S.; Potterton, E. A.; Powell, H. R.; Read, R. J.; Vagin, A.; Wilson, K. S., Overview of the CCP4 suite and current developments. Acta Crystallogr. D Biol. Crystallogr. 2011, 67, (Pt 4), 235-42.

(35) Mysinger, M. M.; Carchia, M.; Irwin, J. J.; Shoichet, B. K., Directory of useful decoys, enhanced (DUD-E): better ligands and decoys for better benchmarking. J. Med. Chem . 2012, 55, (14), 6582-94.

(36) Liu, T.; Lin, Y.; Wen, X.; Jorissen, R. N.; Gilson, M. K., BindingDB: a web-accessible database of experimentally determined protein-ligand binding affinities. Nucleic Acids Res. 2007, 35, (Database issue), D198-201.

(37) Gonzalez, A.; Quirante, J.; Nieto, J.; Almeida, M. R.; Saraiva, M. J.; Planas, A.; Arsequell, G.; Valencia, G., Isatin derivatives, a novel class of transthyretin fibrillogenesis inhibitors. Bioorg. Med. Chem. Lett. 2009, 19, (17), 5270-3.

(38) Choi, S.; Connelly, S.; Reixach, N.; Wilson, I. A.; Kelly, J. W., Chemoselective small molecules that covalently modify one lysine in a non-enzyme protein in plasma. Nat. Chem. Biol. 2010, 6, (2), 133-9.

(39) Gupta, S.; Chhibber, M.; Sinha, S.; Surolia, A., Design of mechanism-based inhibitors of transthyretin amyloidosis: studies with biphenyl ethers and new structural templates. J. Med. Chem. 2007, 50, (23), 558999.

(40) Bento, A. P.; Gaulton, A.; Hersey, A.; Bellis, L. J.; Chambers, J.; Davies, M.; Kruger, F. A.; Light, Y.; Mak, L.; McGlinchey, S.; Nowotka, M.; Papadatos, G.; Santos, R.; Overington, J. P., The ChEMBL bioactivity database: an update. Nucleic Acids Res. 2014, 42, (Database issue), D1083-90.

(41) Julius, R. L.; Farha, O. K.; Chiang, J.; Perry, L. J.; Hawthorne, M. F., Synthesis and evaluation of transthyretin amyloidosis inhibitors containing carborane pharmacophores. Proc. Natl. Acad. Sci. U. S. A. 2007, 104, (12), 4808-13.

(42) Sant'Anna, R.; Gallego, P.; Robinson, L. Z.; Pereira-Henriques, A.; Ferreira, N.; Pinheiro, F.; Esperante, S.; Pallares, I.; Huertas, O.; Rosario Almeida, M.; Reixach, N.; Insa, R.; Velazquez-Campoy, A.; Reverter, 
D.; Reig, N.; Ventura, S., Repositioning tolcapone as a potent inhibitor of transthyretin amyloidogenesis and associated cellular toxicity. Nat. Commun. 2016, 7, 10787.

(43) Cianci, M.; Folli, C.; Zonta, F.; Florio, P.; Berni, R.; Zanotti, G., Structural evidence for asymmetric ligand binding to transthyretin. Acta Crystallogr. D Biol. Crystallogr. 2015, 71, (Pt 8), 1582-92.

(44) Rosen, H. N.; Murrell, J. R.; Liepnieks, J. J.; Benson, M. D.; Cody, V.; Moses, A. C., Threonine for alanine substitution at position 109 of transthyretin differentially alters human transthyretin's affinity for iodothyronines. Endocrinology 1994, 134, (1), 27-34.

(45) Scifinder, Chemical Abstracts Service. http://www.cas.org/products/scifinder (accessed April 8,2016) 2015.

(46) Purkey, H. E.; Dorrell, M. I.; Kelly, J. W., Evaluating the binding selectivity of transthyretin amyloid fibril inhibitors in blood plasma. Proc. Natl. Acad. Sci. U. S. A. 2001, 98, (10), 5566-71.

(47) Hamers, T.; Kamstra, J. H.; Sonneveld, E.; Murk, A. J.; Kester, M. H.; Andersson, P. L.; Legler, J.; Brouwer, A., In vitro profiling of the endocrine-disrupting potency of brominated flame retardants. Toxicol. Sci. 2006, 92, (1), 157-73.

(48) Weiss, J. M.; Andersson, P. L.; Lamoree, M. H.; Leonards, P. E.; van Leeuwen, S. P.; Hamers, T., Competitive binding of poly- and perfluorinated compounds to the thyroid hormone transport protein transthyretin. Toxicol. Sci. 2009, 109, (2), 206-16. 